\title{
Le milieu didactique : d'une étude empirique en contexte difficile à une réflexion sur le concept
}

\section{Chantal Amade-Escot et Patrice Venturini}

\section{OpenEdition}

12 Journals

\section{Édition électronique}

URL : https://journals.openedition.org/educationdidactique/407

DOI : 10.4000/educationdidactique.407

ISBN : 978-2-7535-1620-5

ISSN : 2111-4838

\section{Éditeur}

Presses universitaires de Rennes

\section{Édition imprimée}

Date de publication : 1 mars 2009

Pagination : $7-43$

ISBN : 978-2-7535-0872-9

ISSN : 1956-3485

\section{Référence électronique}

Chantal Amade-Escot et Patrice Venturini, « Le milieu didactique : d'une étude empirique en contexte difficile à une réflexion sur le concept », Éducation et didactique [En ligne], 3-1 | mars 2009, mis en ligne le 01 mars 2011, consulté le 22 juillet 2022. URL : http://journals.openedition.org/

educationdidactique/407; DOI : https://doi.org/10.4000/educationdidactique.407 


\title{
LE MILIEU DIDACTIQUE : D'UNE ÉTUDE EMPIRIQUE EN CONTEXTE DIFFICILE À UNE RÉFLEXION SUR LE CONCEPT
}

\author{
Chantal Amade-Escot, DiDiST-CREFI-T, Paul Sabatier, \\ Patrice Venturini, DiDiST- CREFI-T, Paul Sabatier \\ E TEA, ENFA Université de Toulouse
}

\begin{abstract}
Résumé : Nous poursuivons dans cet article le travail théorique déjà largement entamé en didactique, autour du concept de «milieu». Nous prenons d'abord appui sur une étude empirique concernant la comparaison de séances ordinaires d'enseignement de l'éducation physique et sportive (EPS) et de physique menées par deux professeurs d'un même collège «ambition réussite». Les phénomènes didactiques étant exacerbés dans le contexte d'enseignement difficile, celui-ci permet de mieux saisir certains aspects critiques (décrits dans l'article) des processus mésogénétiques à l'œuvre dans l'action conjointe professeur élèves. Nous revenons ensuite sur les principales conceptualisations de la notion de milieu. Nous avançons l'idée que les points de vue différents qu'elles présentent peuvent être dépassés en considérant la complémentarité des dimensions institutionnelles et situationnelles à l'œuvre. Cette analyse et les résultats de l'étude empirique nous conduisent à envisager au sein de la théorie de l'action didactique conjointe, le milieu comme résultant d'un processus dynamique, généralement différentiel, inscrit dans des échelles temporelles multiples articulant les registres de l'activité et de l'action didactiques.
\end{abstract}

Mots clés : Milieu didactique - Mésogenèse - Institution - Situation - Activité didactique - Action didactique conjointe - Milieu difficile

Chantal Amade-Escot \& Patrice Venturini

Poursuivant le travail de théorisation didactique autour de la notion de milieu tel que développé en didactique (Amade-Escot, 2005, Brousseau, 1990; Orange, 2007; Perrin-Glorian, 1999; Johsua et Félix, 2002; Schubauer-Leoni, 2008; Sensevy et Mercier, 2007), cet article présente les résultats d'une étude visant à comparer les formes de "mise en scène * (Brousseau, 1986) des savoirs au cours de séances ordinaires d'enseignement de l'éducation physique et sportive (EPS) et de la physique en « établissement difficile $^{1} »$. La visée de cette recherche comparative consiste à ré-interroger les présupposés expliquant les difficultés rencontrées par les élèves et les enseignants dans ce type de contexte. Il s'agit de rendre compte, au plus près des dispositifs d'enseignement et d'apprentissage mis en place, des modalités du fonctionnement didactique. En effet, comme nous le discutons par ailleurs (Monnier et Amade-Escot, 2009, Venturini et Amade-Escot, soumis), si de nombreux rapports ont établi les inégalités scolaires persistantes et la faible efficacité des politiques éducatives territorialisées de compensation de ces inégalités, la question des pratiques d'enseignement dans les milieux difficiles reste, comparativement aux analyses économiques et sociologiques, un domaine de recherche peu exploré. Aussi, nous considérons nécessaire et utile de s'intéresser aux formes spécifiques « du » didactique ordinaire (Mercier, Schubauer-Leoni et Sensevy, 2002) en contexte dit « difficile». Nos travaux s'inscrivent délibérément dans une approche considérant que la diffusion des savoirs est au cœur de l'activité enseignante - y compris et plus encore - dans ces contextes. Aussi proposons-nous tout d'abord un point sur la question des pratiques d'enseignement et d'apprentissage qui y sont développées avant de préciser la problématique à laquelle répond notre recherche. Nous décrivons ensuite les caractéristiques de l'environnement scolaire dans lequel nous avons mené l'étude ainsi que la manière dont nous avons recueilli puis traité les données. Nous poursuivons en discutant, à la lumière de quelques extraits de séances, la façon dont les interactions élèves, professeur, savoir sont susceptibles d'expliquer quelques traits de fonctionnement déjà mis en évidence par d'autres recherches. Nous centrons l'analyse sur le milieu, en ce qu'il résulte d'une production conjointe du professeur et des élèves, pour mettre en évidence quelques traits génériques de l'action didactique en contexte difficile tant en EPS qu'en physique, tout en soulignant leur spécificité irréductible. Nous terminons enfin en revenant sur les concepts de milieu et de mesogenèse, leur statut dans les recherches portant sur les classes ordinaires pour en discuter la pertinence et 
l'intérêt dans l'approche comparatiste en didactique ${ }^{2}$. Nous en proposerons une lecture visant à articuler les registres d'analyse de l'activité et de l'action didactiques alliant les points de vue institutionnel et situationnel et leurs temporalités respectives.

\section{Quelques tendances récurrentes des pratiques d'apprentissage et d'enseignement en établissement difficile}

Si peu d'études didactiques ont été conduites en établissement difficile, il existe toutefois un ensemble de travaux s'intéressant pour les uns aux pratiques d'apprentissage des élèves en zone d'éducation prioritaire (ZEP) et pour les autres s'attachant à décrire les formes des pratiques enseignantes. La revue de question effectuée par Rochex et Kherroubi en 2004 en présente une vision assez complète.

Selon les résultats qu'ils rapportent, les élèves des ZEP, essentiellement dans l'action, sont sensibles aux traits de surface des situations éducatives et ont du mal à faire le lien entre celles-ci et les savoirs visés. De plus, les réquisits du travail et de la réussite scolaire restent opaques pour eux (Van Zanten, 2001). De leur coté, les enseignants ont à gérer un certain nombre de contradictions entre une logique d'apprentissage et une logique de socialisation considérée comme préalable et extérieure aux activités disciplinaires, logiques perçues de ce fait comme concurrentes (Rochex $\&$ Kherroubi, 2004). Les injonctions officielles qui pèsent sur l'activité enseignante (Amigues, Félix, \& Saujat, 2008) préconisent une individualisation des activités afin de répondre à l'hétérogénéité du groupe classe. Ces injonctions se traduisent en pratique par un évitement des phases collectives certes difficiles à gérer sur le plan disciplinaire, mais qui a pour conséquence un affaiblissement dans la construction de références communes à l'ensemble de la classe (Bautier, 1992 ; Debars \& Amade-Escot, 2006; Peltier-Barbier, 2004). Lorsqu'il a lieu, le travail collectif concerne l'introduction de nouveaux savoirs, il est mené le plus souvent sur le mode maïeutique (Butlen, Barbier-Peltier, \& Pezard, 2002; Peltier-Barbier, 2004). D’une manière générale, les auteurs s'accordent pour dire que les enseignants s'attachent à favoriser l'engagement des élèves dans les apprentissages en mettant en place des situations permettant une réussite immédiate, ce qui se traduit par une adaptation des objectifs et des stratégies au niveau supposé des élèves. Les tâches proposées, généralement fermées, sont souvent très décontextualisées et simplifiées. L'étude des savoirs est étroitement pilotée par les consignes de l'enseignant, l'oral y est privilégié (Bautier, 1992; Bautier \& Goigoux, 2004; Peltier-Barbier, 2004, Debars \& Amade-Escot, 2006, Van Zanten, 2001). Ce sur-ajustement didactique aux caractéristiques supposées des élèves a des effets cumulatifs dans le temps, d'autant plus que le travail personnel exigé est le plus souvent limité à la classe.

Finalement, la plupart des auteurs qui se sont intéressés aux pratiques effectives d'enseignement et d'apprentissage en contexte dit difficile pointent des gestes professoraux se traduisant par un éclatement et une réduction des contenus enseignés sous forme de micro-tâches très individualisées et une absence de phase collective d'institutionnalisation des savoirs, à l'origine de malentendus sur les enjeux réels des tâches (Bautier, 1992).

\section{Revisiter les formes conjointes de l'enseignement et de l'apprentissage en contexte difficile}

L'ensemble de ces constats accrédite l'idée que les logiques d'action des élèves et des enseignants en contexte difficile se renforcent l'une l'autre et conduisent à un fonctionnement didactique caractérisé par des situations de faible épaisseur épistémologique. Il s'agirait pour les enseignants de maintenir coûte que coûte la relation didactique en créant des conditions d'enrôlement des élèves dans des tâches peu exigeantes relativement aux savoirs visés. Ces constats pessimistes ont ouvert la voie à des études plus nuancées, visant à mieux comprendre les contraintes qui pèsent sur les pratiques enseignantes en contexte difficile, comme en témoignent de récents travaux (Monnier \& Amade-Escot, 2009; Venturini \& Amade-Escot, soumis). Leurs résultats accréditent l'idée, déjà suggérée par Rochex (1997), selon laquelle ces milieux donnent à voir - par un effet de loupe - des phénomènes didactiques plus génériques, dont certains ont été mis en évidence dans les classes plus ordinaires comme l'existence, au cours du processus d'enseignement-apprentissage, d'ajustements plus ou moins maîtrisés, plus ou moins adaptés aux aléas et aux dysfonctionnements inévi- 
tables (même minimes) liés au caractère conjoint de l'action didactique (Amade-Escot, 2002).

C'est pourquoi il nous a paru intéressant d'examiner la co-construction du milieu didactique en contexte difficile, tout en centrant cette étude non pas seulement sur les activités d'apprentissage des élèves, ou sur celle de l'enseignant, mais en la focalisant sur les dimensions conjointes de l'action du professeur et des élèves (Schubauer-Leoni, 2008; Sensevy \& Mercier, 2007) en ce qu'elle est susceptible de mettre en évidence les dynamiques qui président aux constats préalablement évoqués. Nous avons de plus considéré que l'examen comparatiste (Joshua, 2002; Mercier, Schubauer-Leoni \& Sensevy, 2002) d'activités conduites dans un même contexte mais contrastées selon les disciplines en jeu pourrait être riche d'enseignements grâce à l'éclairage réciproque qu'il rend possible, lequel est susceptible de faire apparaître des caractéristiques génériques du processus mésogénétique sans perdre de vue celles qui relèvent des spécificités disciplinaires. Nous pensons en effet que c'est dans le travail solidaire des dimensions génériques et spécifiques que l'on peut construire l'intelligibilité des processus didactiques (Amade-Escot, 2007).

L'enseignement de l'EPS et de la physique au collège nous a semblé propice à l'investigation envisagée dans la mesure où les programmes officiels de ces deux disciplines recommandent de partir de situations problématiques. En EPS, les orientations officielles préconisent de confronter l'élève à des activités physiques, sportives et artistiques (APSA) lui permettant de "s'éprouver physiquement " tout en l'impliquant « dans [des] procédures de compréhension de ses actions » (MEN, 1998). En physique, les élèves doivent résoudre les problèmes posés en mettant en œuvre une démarche d'investigation (MEN, 2005). Il reste que les dispositifs et les matériels utilisés sont spécifiques à chacune des disciplines, tout comme le sont les savoirs transmis, de nature différente. En EPS, ils ont généralement un caractère technique au sens développé par Mauss (1935) à propos des techniques du corps, alors qu'en physique, il s'agit de modèles généralement représentés de manière symbolique.

Les constats précédemment rapportés soulignant un affaiblissement des contenus enseignés en contexte difficile, il nous est apparu pertinent, dans le cadre d'une approche comparatiste, de nous intéresser à des enseignants manifestant explicitement leur volonté de transmettre des savoirs et confrontés de ce fait, à l'ensemble des contraintes du contexte d'exercice qu'en tant que chercheurs nous souhaitions mettre au jour. En résumé, cette étude s'attache à comparer des pratiques didactiques ordinaires en EPS et en physique, telles qu'elles sont menées dans deux classes d'un même collège par des enseignants déclarant vouloir transmettre les contenus disciplinaires selon les recommandations du programme. La recherche s'inscrit dans une approche ascendante de la transposition didactique (Schubauer-Leoni, 2008; Schubauer-Leoni \& Leutenegger, 2005) et cherche à analyser pour la comparer, la co-construction de la référence en classe d'EPS et de physique par les élèves et leur professeur, en s'intéressant plus particulièrement aux processus conjoints de construction et d'évolution des milieux pour l'étude ${ }^{3}$.

\section{Méthodologie}

Pour mener à bien cette comparaison, nous avons mené des investigations dans un collège toulousain appartenant à un "Réseau Ambition Réussite ${ }^{4}$ » avec la collaboration de deux enseignants. Après avoir précisé quelques-unes de leurs caractéristiques pour situer le cadre dans lequel cette recherche a été menée, nous nous intéresserons au recueil des données et à leur traitement.

\section{Le cadre général}

Le collège concerné est considéré comme un des plus difficiles de l'agglomération toulousaine. Aux dires des enseignants dont le renouvellement est important, les agressions physiques entre élèves sont régulières, les agressions verbales en classe et les vols fréquents, la violence est quotidienne. L'enseignant d'EPS observé a 32 ans d'ancienneté dont 10 dans ce collège. Il se considère comme « un militant de l'éducation physique ». La classe de $6^{\mathrm{e}}$ observée est selon lui une classe difficile dans laquelle les 15 élèves causent du désordre: "les garçons surtout font preuve de vulgarité, mais aussi les filles». Il a aussi fallu y canaliser quelques réactions racistes depuis le début de l'année. Toutefois les élèves sont, 
toujours selon lui, dynamiques, indisciplinés mais motivés ( il y a des élèves qui perturbent les autres qui se laissent entraîner "; "ces élèves, ils ont de bonnes capacités physiques ", entretien ante). L'enseignante de physique a quant à elle, 10 ans d'ancienneté dont neuf passés dans diverses ZEP de l'hexagone. Elle est très attachée à son métier et malgré les conditions difficiles, l'exerce toujours avec plaisir. Elle dit être bien installée dans la classe de $5^{\mathrm{e}}$ qui a servi de support à l'étude, qu'elle décrit comme ayant "une bonne dynamique »: les 17 élèves sont tout à fait volontaires pour participer oralement aux activités et expérimenter. Ils ont cependant de nombreuses difficultés (dix sont en échec) principalement vis-à-vis de l'écrit et travaillent très peu à la maison. Comme son collègue, l'enseignante considère que la gestion de cette classe reste un problème de tous les instants. Enfin, les deux enseignants ont en commun de chercher à se mettre en conformité avec les recommandations des programmes officiels.

\section{Le recueil des données}

Nous nous sommes fortement inspirés de la méthodologie proposée par Leutenegger (2003). Considérant l'apprentissage comme résultant d'un interactionnisme social mettant en jeu l'élève, l'enseignant et l'objet enseigné (ou appris), le protocole de recueil de données croise, en les articulant, des informations issues de corpus relatifs aux différents éléments de cette triade afin de valider une hypothèse émise à partir de l'un d'entre eux. Dans cette perspective, nous avons recueilli des données relatives :

- À l'activité des enseignants et des élèves en classe. Nous avons filmé trois séances consécutives d'un cycle de badminton ${ }^{5}$ portant sur le thème du programme « assurer la continuité du jeu et construire, en position favorable, la rupture de l'échange " en « entrant dans l'activité par la gestion d'une cible à libérer puis à exploiter ${ }^{6}{ }_{\gg}$ (cf. MEN, 1998, document d'accompagnement $6^{\text {e }}$, p. 92). Pour ce qui est de la physique, nous avons filmé trois séances consécutives menées en classe dédoublée puis en classe entière ${ }^{7}$, portant sur le thème « Sources de lumière, objets diffusants, condition de visibilité d'un objet ». Celui-ci, qui débute l'optique en $5^{\text {e }}(\mathrm{MEN}, 2005)$ traite : i) des types de sources, primaires et secondaires, respectivement caractérisés par l'émission et la diffusion de la lumière; ii) de la vision, possible seulement si la lumière émise ou diffusée pénètre dans l'œil.

- Aux documents utilisés dans les séances (fiches de travail données aux élèves), aux programmes officiels du collège et à leurs documents d'accompagnement. L'ensemble permet de repérer les savoirs, enjeux didactiques des séances observées, et les démarches préconisées pour les transmettre.

- Aux intentions didactiques et aux analyses a posteriori des enseignants quant aux séances qu'ils ont conduites. Des entretiens ante- et post- séances ont été réalisés avec eux pour cerner leurs objectifs et obtenir une première analyse à chaud de leur activité.

\section{Le traitement des données}

Les vidéos de séances constituent le corpus principal. Dans un premier temps, nous avons condensé ces données en établissant le synopsis de chaque séance (Leutenegger, 2003) permettant de mettre en relation un repérage temporel, les différentes tâches et leurs visées, les ressources disponibles pour leur réalisation. Pour chacune des tâches, nous avons décrit les modalités de travail et détaillé ce que disent et font enseignant et élèves. Cette première étape d'analyse a permis de faire apparaître un certain nombre d'épisodes ou d'évènements remarquables (au sens de Leutenegger, 2003). Parmi eux, sans nous interdire de faire référence à d'autres pièces du corpus, nous avons retenu pour cet article quatre épisodes susceptibles d'éclairer la manière dont l'action conjointe des professeurs et des élèves contribue à la construction du milieu didactique :

- Deux épisodes en EPS, observés lors de la seconde séance dont le thème est : «pourquoi et comment construire un dégagé donnant du temps?". Pour les élèves, il s'agit d'identifier les conditions de production d'une frappe de dégagement ${ }^{8}$ leur permettant de prendre des informations sur la position de l'adversaire, de le déplacer, afin d'être en situation de marquer le point (ce dernier enjeu est mis en perspective mais n'est pas réellement travaillé dans la séance). Le premier épisode (min. 10 à 28) porte sur des enjeux de savoirs imbriqués, à la fois techniques et tactiques : amélioration de la posture de frappe à 
main haute pour pouvoir produire des trajectoires de volant en hauteur, prise d'information visuelle sur le placement de l'adversaire. Les élèves par deux, sont répartis sur sept terrains ${ }^{9}$ de badminton et travaillent en autonomie. L'enseignant passe de groupe en groupe. Le second épisode (min. 29 à 31) concerne l'élaboration collective, par la classe réunie au milieu du gymnase, des raisons et de la manière d'effectuer une frappe de dégagement. Il met en scène des éléments prototypiques de la façon dont l'enseignant d'EPS conduit les interactions verbales avec les élèves. Ces deux épisodes successifs se situent dans la première moitié de la séance après un moment de prise en main de la classe (6 minutes) visant à obtenir l'attention des élèves et à présenter le travail, suivi de 4 minutes d'échauffement. La deuxième moitié de séance se poursuit par une brève reprise de la tâche du premier épisode, suivi de deux courts moments de matchs : le premier revenant sur le travail de séance précédente, le second visant l'application de la frappe de dégagement étudiée.

- Deux épisodes en physique, portant sur deux situations problématiques lors de la séance en classe entière. Cette dernière se situe après les deux séances en demi-classe qui ont permis d'introduire la distinction entre les sources primaires (production de la lumière) et les sources secondaires (diffusion d'une partie de la lumière reçue). Le premier épisode (min. 12 à 26) concerne les conditions de visibilité d'une source primaire, construites à partir d'une expérience : "ce n'est pas parce qu'il y a une lampe dans une salle $q u$ 'on la voit forcément. Quelles conditions doivent être réunies pour que tu puisses voir une lampe? " (cf. fiche élève). Le second, complémentaire (min. 36-49), s'attache à la façon dont l'enseignante conduit les débats avec les élèves autour d'une expérience visant à modéliser le rôle des objets diffusants : "pourquoi voit-on les objets autour de nous alors que souvent ils ne sont pas directement éclairés par une source primaire? " (cf. fiche élève). Ces deux épisodes contribuent (avec d'autres ${ }^{10}$ ) à modéliser la vision par l'œil, ce qui est un enjeu didactique du programme de $5^{\text {e }}$. Dans la séance, ils sont séparés par un épisode (min. 27 à 35) dans lequel les élèves lisent à haute voix la biographie (simplifiée) d'Al-Azhem, scientifique arabe ayant entre autre " modélisé » aux alentours de l'an 1000 le principe de la vision des objets. Toutefois, les informations apportées sur ce sujet n'ont pas été réinvesties dans la suite de la séance. Le texte a en effet donné lieu à des questions à traiter à l'extérieur de la classe. Ces trois épisodes ont constitué la partie principale de séance en classe entière, débutée par la réalisation d'exercices (min. 2 à 12).

Dans ces quatre épisodes, la référence relève, en accord les programmes d'une pratique sociale identifiée : pratique de loisir sportif dans un cas (comme énoncé par l'enseignant lors de l'entretien ante); pratique domestique dans l'autre (comme en témoignent les dispositifs expérimentaux et les exemples donnés au cours la séance). Soulignons dans les deux cas, que l'ambition des enseignants est de ne pas réduire le savoir visé à quelques compétences ou savoir faire de bas-niveau.

Pour interpréter les données vidéo, d'autres éléments ont été convoqués. Nous avons analysé, du point de vue de la nature des savoirs et des modalités de leur transmission, les programmes officiels et les documents d'accompagnement relatifs au badminton en classe de $6^{e}$ et à l'optique en classe de $5^{e}$. Cette analyse a permis d'identifier les enjeux de savoir et la manière de les traiter, tels que fixés par l'institution, ainsi que les difficultés qu'ils recèlent afin d'éclairer les situations observées. Les données du corpus secondaire ont aussi fait l'objet d'un traitement. Ainsi, de la retranscription verbatim des entretiens anteet post-séances, nous avons extrait les propos qui donnent du sens, selon nous, à certains évènements repérés lors du traitement de la vidéo. Le contenu des fiches distribuées en physique et la démarche qu'elles induisent ont été examinés au regard des programmes de $5^{\text {e }}$. Les tâches proposées aux élèves dans les séances ont fait l'objet d'une analyse a priori permettant de situer parmi les possibles les comportements observés.

\section{Résultats : une gestion critique des milieux didactiques}

L'ensemble de ces analyses a permis de comparer les processus mésogénétiques en EPS et en physique. Nous discutons dans une première section des conditions d'existence d'un milieu didactique en relation avec les spécificités des deux disciplines; dans la seconde, nous examinons la manière (générique) dont les enseignants conduisent les interactions verbales constitutives de ces processus. Ces deux 
analyses nous conduiront à conclure sur la difficulté à créer une dynamique mésogénétique productive en contexte « difficile».

Compte tenu de la taille de l'article, nous avons choisi d'exemplifier à chaque fois ces analyses à partir des traits les plus saillants de l'action des enseignants en ce qu'elle vient s'articuler à celle des élèves. Ce point de vue, déjà avancé dans la problématique, ne sera pas discuté ici. Nous reviendrons en effet dans la dernière partie de l'article sur les différentes conceptualisations proposées pour la notion de milieu, dont celle de l'action didactique conjointe, utilisée ici.

\section{Conditions d'existence d'un milieu didactique : spécificités disciplinaires en EPS et en physique}

Dans cette section nous nous attachons donc à pointer les conditions spécifiques qui permettent à un dispositif de « faire milieu ». En effet, si comme l'indiquent Schubauer-Leoni, Leutenegger, Ligozat et Flückiger (2007) à propos de l'étude « du didactique ordinaire », les dispositifs initiaux, construits et proposés par l'enseignant sont potentiellement pertinents pour l'apprentissage des savoirs visés, il reste toutefois « à charge de la recherche d'en établir la portée didactique ", c'est-à-dire à mettre en évidence en quoi et à quelles conditions ils peuvent «faire milieu » (p. 56). Nous nous intéressons successivement à deux épisodes. Le premier en EPS rend compte des difficultés rencontrées par l'enseignant pour créer un milieu vif ${ }^{11}$, le second en physique met en évidence comment l'enseignante réussit en délimitant très finement les possibles et les interdits, à structurer avec ses élèves un milieu a priori propice à la rencontre de la modélisation envisagée. Pour rendre compte des observations, nous présentons pour chaque discipline les enjeux de la séance dans laquelle l'épisode analysé prend place, puis nous rendons compte de l'avancée ou de l'échec du processus mésogénétique conjoint. Cela nous amène, en conclusion, à considérer le milieu comme une dimension problématique de l'action didactique conjointe.

\section{Enjeux didactiques de la séance de badminton}

L'entretien ante et le synopsis de la séance indiquent que l'enseignant souhaite amener les élèves à identifier les conditions d'utilisation d'un coup de dégagement permettant de déplacer l'adversaire latéralement, en conformité avec les programmes (assurer la continuité du jeu et libérer une cible à attaquer, MEN, 1998). Les difficultés rencontrées par les élèves sont liées au fait que dans une même frappe du volant, il s'agit à la fois de défendre son camp et d'attaquer le camp adverse. Cette simultanéité impose aux élèves de différencier des coups visant à ralentir le jeu pour se donner le temps de construire le point, de ceux visant à marquer le point (Gomet, 2003). Dans la séance observée, l'enseignant cherche à amener les élèves à ralentir le jeu pour, à la fois, se donner le temps de la prise d'information sur l'adversaire, l'obliger à se déplacer et améliorer la frappe haute du volant. Différentes prises d'information sont nécessaires : sur le placement de l'adversaire afin de le déplacer; sur la trajectoire du volant qui arrive pour assurer la rencontre tamis/volant. La réalisation d'un coup de dégagement suppose une frappe du volant légèrement en arrière, au-dessus de la tête, tamis orienté vers le haut. Elle implique une réorganisation posturale à laquelle font obstacle les usages de manipulation ordinaire des objets (Marsenach, 1995). En effet, le plus souvent les frappes initiées par des élèves de $6^{\mathrm{e}}$ sont dites « en poussette », car ils valorisent la recherche de la rencontre tamis/volant, ce qui les amène à effectuer des frappes devant le visage, bras fléchis, ne permettant pas de varier les trajectoires ni en hauteur ni en direction. Les volants sont de ce fait renvoyés de l'autre coté du filet dans la zone centrale du terrain adverse, ce qui ne crée pas les conditions du déplacement recherché de l'adversaire et rend donc caduque la nécessité d'une prise d'information sur son placement (Roustan, 2003).

\section{Difficulté de faire vivre un milieu didactique en EPS}

Nous discutons ici les raisons qui font que l'enseignant d'EPS est contraint et ne peut faire avancer le savoir parce que le dispositif initialement conçu pour faire vivre aux élèves l'expérience du déplacement du joueur adverse ne peut remplir son but.

L'épisode (min. 10 à 28) constitue le corps de la séance. L'enseignant met en place une tâche d'échanges de volant (un jeu de duo) dans laquelle un élève doit obliger le joueur-partenaire situé de l'autre coté du filet à se déplacer dans le sens de la 
largeur. Cette tâche peut être considérée comme une situation problématique pertinente par rapport aux savoirs visés (produire un coup de dégagement pour déplacer l'adversaire latéralement). L'intention didactique vise un enjeu à plus long terme : le déplacement latéral de l'adversaire libérera un espace pour marquer le point lorsqu'on passera d'un jeu de duo à un jeu d'opposition (en fin de séance) : "c'est-à-dire que si vous tirez sur lui vous vous êtes aperçu que vous ne marquez pas de point, maintenant il va falloir essayer de le faire courir un tout petit peu d'accord " (min. 4 lors de la présentation de la séance). Pour faire vivre aux élèves l'expérience du déplacement du joueurpartenaire, l'enseignant propose un terrain agencé pour faciliter la tâche du joueur qui déplace son adversaire : le premier joue sur un terrain normal, le second doit protéger un espace plus large puisque son terrain comprend aussi les couloirs latéraux de $45 \mathrm{~cm}$ utilisés « en double ».

L'analyse a priori de cette tâche met en évidence deux enjeux de savoir emboîtés : l'affinement de la frappe du volant pour produire un « dégagement défensif de précision » (reprise du travail technique de la séance précédente abordant le dégagement latéral) et la prise d'information (pour envoyer le volant à droite, à gauche) sur un terrain élargi. Cette dernière variable pourrait être pertinente, mais réussir le jeu d'échanges suppose de produire des trajectoires $\mathrm{du}$ volant en hauteur et en direction ${ }^{12}$ (conditions nécessaires pour donner le temps du déplacement et/ou du repositionnement aux deux joueurs). Or, de telles trajectoires sont d'autant plus difficiles à produire que les élèves frappent le volant en déséquilibre. L'agencement du dispositif ne suffit pas à créer des conditions susceptibles de favoriser ce double enjeu car les retours du volant par le joueur qui a été déplacé risquent d'être très aléatoires. La continuité des échanges visée par ce jeu de duo ne peut pas être garantie par le dispositif ${ }^{13}$. En effet, si l'un des deux joueurs ne parvient pas à renvoyer le volant suffisamment haut pour donner au partenaire (et à lui-même) le temps du replacement, le milieu didactique s'évanouit. Ainsi, dès la mise en place de la tâche se trouve donc posée la question de la possibilité d'existence d'un milieu didactique puisque les élèves par leurs actions doivent contribuer à le faire vivre. La création a priori d'un milieu antagoniste ${ }^{14}$ (Brousseau, 1990) est donc ici critique, mais aussi plus généralement en EPS (Amade-Escot, 2005). Dans cette séance de badminton, l'aspect problématique de la tâche porte autant sur les savoirs mis à l'étude que sur les conditions à réunir pour qu'elle « fasse milieu » pour les élèves. Ce dernier point est d'autant plus délicat qu'il faut éviter, dans ce contexte, que leurs échecs entraînent une désaffection. Les stratégies de l'enseignant, son jeu sur le jeu des élèves (au sens de la théorie de l'action conjointe en didactique, Schubauer-Leoni et al. 2007 ; Sensevy \& Mercier, 2007) vont tenter de réduire cet écart :

- L'enseignant consacre tout d'abord six minutes à définir le jeu didactique en s'appuyant sur deux élèves pour montrer ce qu'il y à faire. Puis les élèves sont invités à travailler sur les sept terrains du gymnase. L'enseignant passe de groupe en groupe (min. 16 à 28) pour rappeler la consigne initiale ( "tu joues en hauteur, en hauteur»). Ces rappels sont ponctués de démonstrations (comment produire une trajectoire en hauteur) et d'injonctions vives auprès de sept élèves qui traînent où qui discutent. À la 20 ème minute tous les élèves sont au travail, et ce malgré un incident disciplinaire (vite réglé) avec Farid, l'un d'entre eux.

- Une fois l'activité des élèves lancée, la vidéo met en évidence très peu de continuité dans les échanges qui se limitent à deux ou trois frappes successives. Seuls quelques élèves réussissent à échanger le volant, notamment en ne déplaçant plus leur camarade de jeu.

- Dans cet épisode, toute l'action régulatrice du professeur consiste à rappeler les conditions favorables à l'échange de volant afin de faire vivre un possible milieu pour l'étude. Il privilégie les consignes centrant les élèves sur le temps que l'on se donne quand on joue le volant. Passant auprès des élèves sur chaque terrain de badminton, il valorise les trajectoires hautes : "ça, j'appelle ça en hauteur, quand tu joues en hauteur, tu te donnes du temps à toi pour te déplacer» (min. 23).

- Pendant les 12 minutes d'activité des élèves, il passe de groupe en groupe, insiste sur la hauteur donnée au volant. On note 19 occurrences d'injonctions auprès des élèves pendant l'épisode (" haut, haut! "; "je vais, je reviens, je frappe en hauteur "; "on a dit on joue en hauteur", etc.). La présence physique de cet enseignant tour à tour auprès de 


\section{LE MILIEU DIDACTIQUE : D'UNE ÉTUDE EMPIRIQUE EN CONTEXTE DIFFICILE À UNE RÉFLEXION SUR LE CONCEPT}

Chantal Amade-Escot \& Patrice Venturini

chacun des élèves témoigne de son souci de tenir sa classe mais aussi de s'occuper de chacun d'entre eux : « essayer d'expliquer individuellement, ils [les élèves de cette classe] ont besoin de réponses individuelles"; " dès que l'on parle au groupe c'est un peu l'opposition entre eux" (entretien ante).

- Les échecs amènent les élèves à se déplacer pour ramasser les volants par terre, ils provoquent la désaffectation de garçons que l'enseignant remet rapidement en activité (min. 23). Très impliqué, restant au plus près de chacun en passant de terrain en terrain, ses interventions verbales continues combinent des injonctions sur la hauteur à donner au volant et sur la réflexion à produire: "plus en hauteur sinon on peut pas faire l'exercice "; "Soraya, comment tu étais ... essaie de réfléchir "; " Khaled, par où il faut le taper si tu veux qu'il aille en hauteur? ».

- Cependant au fil de l'exercice, la question initiale (déplacer l'adversaire) semble progressivement être perdue de vue, au moins dans le temps d'accompagnement par l'enseignant de l'activité des élèves dans ce jeu de duo.

L'interprétation de l'épisode confirme que la tâche installée par l'enseignant ne peut «faire milieu » que lorsque les trajectoires sont assez hautes pour donner du temps aux deux protagonistes. Pour cela, ils doivent frapper le volant au dessus et en arrière du plan de la tête et ainsi produire le « dégagé » du volant en hauteur. La condition de possibilité de ce jeu d'apprentissage (Sensevy, 2007) suppose la construction d'une posture de frappe. Dans l'impossibilité d'obtenir des comportements moteurs favorables à la production de ce type de trajectoire, et malgré toutes les injonctions à monter le volant en hauteur, le temps didactique stagne, ce qui amène l'enseignant à réduire la visée de la tâche et à laisser tomber la question du déplacement du joueur (enjeu tactique essentiel qui confère le sens même de ce type d'action en badminton : cf. analyse a priori). En fait, la tâche ne peut «faire milieu » pour l'étude des savoirs visés, car l'existence d'un milieu didactique reste très dépendante des réalisations des élèves. Ce point rend compte de l'incertitude qui pèse sur la création d'un milieu antagoniste (au sens de Brousseau, 1990) ou de la difficulté à en contrôler les « trous » (ou « les ignorances ») à combler (au sens de Johsua \& Félix, 2002). Ici, la spécificité disciplinaire engage l'enseignant dans une quête des conditions qui pourront permettre aux élèves de vivre l'expérience d'un échange de volant compatible avec les enjeux de savoirs visés. Or, les élèves sont encore loin de pouvoir déplacer leur adversaire - enjeu tactique de la séance - et le professeur ne peut compter sur la continuité de l'échange pour que les élèves en éprouvent la pertinence.

\section{Enjeux didactiques de la séance de physique}

La séance présentée dans cet article participe à la construction d'un modèle scientifique de la vision, en s'appuyant sur la caractérisation des sources primaires et secondaires effectuée dans les séances en demiclasse. En effet, selon les deux conceptions très largement dominantes à cet âge (Andersson \& Karrqvist, 1983; Guesne, 1983; La Rosa et al., 1984; Selley, 1996; Tiberghien, 1983), la lumière n'intervient que pour constituer un «bain » général dans lequel trempe l'objet, ou bien elle éclaire simplement l'objet, ce qui permet alors de le voir. Dans les deux cas, si la lumière est nécessaire à la vision, elle ne se propage pas jusqu'à l'œil. Le modèle du physicien qui met en jeu la réception d'un rayonnement par l'œil, qu'il soit émis par une source primaire ou qu'il soit diffusé par une source secondaire, est pratiquement inexistant, et la propriété d'émettre de la lumière est clairement et seulement attribuée à quelques objets (lampes, feu, soleil...). L'appréhension de ce modèle constitue donc un enjeu de taille pour des élèves de cet âge.

Une fiche distribuée aux élèves lors de la séance précédente en demi-classe récapitule l'ensemble des étapes de cette séquence sur la vision et les différentes questions et expériences qui en structurent la chronogenèse. La structuration des différents épisodes souligne d'ailleurs l'ambition de l'enseignante à l'égard des savoirs disciplinaires et son souci du respect des exigences du programme quant à la démarche mise en œuvre. Leur organisation est pratiquement identique : i) situation-problème initiée par une observation ou une question; ii) réflexion collective permettant l'émission et la confrontation d'hypothèses liées aux conceptions des élèves, contribuant à l'appropriation de la question et à la préparation de l'expérience; iii) expérience (réalisée dans cette séquence par l'enseignante) au cours de laquelle une première description du phénomène et son interprétation sont élaborées en commun; iv) formalisation individuelle, à la charge 
de l'élève, de ces observations et interprétations; v) présentation par un élève de ces éléments formalisés, discussion collective puis institutionnalisation systématique dictée par l'enseignante (ou parfois un élève) du texte complétant la fiche élève.

L'action de l'enseignante: délimitation du milieu didactique

L'extrait 1 présenté ci-après est caractéristique de la manière dont l'enseignante procède avec ses élèves. Il s'agit ici, dans le cadre d'une expérience de "pensée ", conduite dans la classe éclairée, de travailler sur les conditions de visibilité d'une source primaire (émission de lumière se propageant jusqu'à l'œil) pour préparer l'expérience réelle qui suivra dans l'obscurité. Pour cela, l'enseignante s'appuie sur les objets posés sur la paillasse : un générateur, un interrupteur, une lampe à incandescence, un écran opaque. Montrant la lampe du doigt (cette dernière pouvant être allumée ou éteinte) ainsi que l'écran opaque, elle entend faire préciser aux élèves, les conditions dans lesquelles soit elle, soit les élèves voient ou ne voient pas la source primaire.

(1) P : Quelles conditions doivent être réunies pour que je puisse voir une lampe? Pour ça... il faut du matériel, j'utilise la lampe, je vais utiliser un générateur, enfin une pile, j’ai la possibilité d'éteindre ou d'allumer la lampe avec l'interrupteur, et j'utilise un écran opaque. Opaque c'est le contraire de transparent El : opaque?

Suivent quelques échanges non rapportés sur le sens de l'adjectif opaque P : (pour clôturer les échanges) C'est un écran opaque, c'est un écran qui ne laisse pas passer la lumière Bon, Vous lisez les consignes ... (les élèves lisent, $P$ fait quelques rappels à l'ordre) d'abord donc, comment faire pour que vous ne puissiez pas voir la lampe et moi non plus? Je suis de ce côté du bureau (le matériel est sur le bureau), vous êtes de l'autre, tout à l'heure, je vais éteindre (la lampe de la salle), comment je peux faire avec ce matériel pour vous empêcher de voir la lampe?

(4) Ho: on va l'éteindre

(5) P : je ne dois pas la voir non plus moi

(6) Ho : on éteint !
(7) P : On éteint

(8) Elèves : quelques propositions peu audibles

(9) P : Si je n'allume pas la lampe est ce que (montrant le matériel) est ce que les conditions sont réunies là? Est ce que vous, vous la voyez pas et est ce que moi je ne la vois pas?

(10) Ho : Oui

(11) P : Si elle est éteinte ça peut marcher

(12) Ho : Oui

(13) $\quad P$ : Bien. Pour que je ne voie plus la lampe mais que vous vous la voyiez toujours, qu'est ce qu'il faudrait que je fasse?

(14) El : la tourner de l'autre côté

(15) P : Donc il faudrait que je l'allume

(16) El : Et qu'elle soit tournée de l'autre côté

(17) El : Qu'on mette l'opaque devant la lampe

(18) P : Ah que je mette l'écran opaque devant. Si je mets l'écran opaque ici, qu'est ce qui se passe au niveau de la lumière? comment se fait il que je ne vois plus la lampe?

(19) Ho : parce que il y a le truc opaque

(20) P : Et alors? Avec la lumière?

(21) Ho : ben, c'est pas transparent

(22) El : Ben ça...

(23) El : Ça renvoie

(24) P : Ça renvoie? L'écran?

(25) El : la lumière

(26) P : Je vous ai dit tout à l'heure que opaque c'était le contraire de transparent, donc ça veut dire que ça ne laisse pas passer la lumière, donc la lumière effectivement, qui est envoyée par la lampe, dans la mesure où j'ai cet écran cette lumière ne peut pas traverser cet écran, et donc il est impossible que je la vois.

Extrait 1 : Classe entière en physique min 12 à 15, délimitation du milieu et démarche maïeutique.

$(P=$ Professeur,$E l=$ Elèves non identifiés, $H o=$ Housseme $)$

La question posée dans la fiche de travail distribuée aux élèves (" quelles sont les conditions que je dois réunir pour arriver à voir une source primaire de lumière ») est associée d'emblée au tour de parole 1 (tdp 1) à un ensemble matériel désigné aux élèves et définissant ainsi les grands contours matériels du milieu : lampe, écran, interrupteur. Les caractéristiques de ces objets qui sont pertinentes pour l'étude sont précisées, contribuant ainsi à une délimitation plus précise du milieu : possibilité d'allumer et d'éteindre la lampe, en relation avec l'émission 


\section{LE MILIEU DIDACTIQUE : D'UNE ÉTUDE EMPIRIQUE EN CONTEXTE DIFFICILE À UNE RÉFLEXION SUR LE CONCEPT}

de lumière (tdp 1), écran ne laissant pas passer la lumière, en relation avec la propagation de cette dernière jusqu'à l'œil (tdp 3). Une première question (tdp 3) invite les élèves à réfléchir sur la nécessité de l'émission de lumière par la source pour que celle-ci soit perçue : «comment faire pour que vous ne puissiez pas voir la lampe et moi non plus? ». Une autre question traitée dans un second temps doit les conduire à percevoir la nécessité pour la lumière de parvenir jusqu'à l'œil : " pour que je ne vois plus la lampe mais que vous vous la voyiez toujours, qu'est ce qu'il faudrait que je fasse? " (tdp 13). Les élèves raisonnent ainsi à chaque fois sur un milieu « réduit» par les questions de l'enseignante, en lien avec chaque paramètre du phénomène ${ }^{15}$. En même temps, elle positionne dans les échanges les éléments importants pour le raisonnement. Ainsi, au tour de parole 15, sa précision " donc, il faudrait que je l'allume ", pointe implicitement aux élèves la nécessité d'une émission de lumière par la source pour qu'elle soit vue. Dans les tours de parole 18 et 20 , elle leur indique par la question qu'elle pose, les éléments conceptuels ou factuels à utiliser dans le raisonnement qui doit aboutir à la nécessité d'une propagation de la lumière jusqu'à l'œil pour assurer la vision de la source.

De manière régulière tout au long de son cours, l'enseignante procède ainsi. Elle délimite avec soin le milieu de l'étude en cours, tant pour ses aspects matériels que conceptuels, puisqu'elle pointe dans tous les cas les éléments sur lesquels ou avec lesquels les élèves sont appelés à raisonner. Dans tous les cas, elle « essaie d'aller à l'essentiel » (et d'autant plus ici qu'elle avance dans la séance), ce que d'ailleurs elle "ne ferait pas dans une classe normale". C'est que dans ce contexte scolaire, il faut selon l'enseignante "faire le maximum en classe", parce qu'en dehors d'une élève (Mélina qui apparaît dans les extraits 2 et 5), les autres ne font rien chez eux ou presque, «ils vivent sur l'instant»(entretien post).

Toutefois, cette délimitation du milieu trouve des limites, perceptibles par exemple dans l'extrait 2 ci-après, au cours duquel l'enseignante expérimente devant les élèves pour tester la validité des différentes prévisions que ceux-ci ont réalisées préalablement avec son aide (cf. extrait 1 ).

(1) P : Inversement quand on vous demande vous de ne plus voir la lumière,
(2) El : On peut fermer les yeux

(3) P : Soit tu fermes les yeux ... c'est valable ... soit on met un écran opaque (P. met l'écran entre les élèves et la lampe)

(4) Ka : on la voit la lampe (Ka perçoit en fait la lumière diffusée par les objets alentour, éclairés par la lampe)

(5) $\mathrm{P}: \mathrm{Tu}$ vois pas la lampe,

(6) Ka : si je la vois

(7) P:Tu ne vois pas la lampe,

(8) Mé : Non

(9) Ka : si on la voit

(10) P : je te parle pas de la lumière, je te parle de la lampe... Kader?

(11) Ka : Ouiii

(12) P : ... la lampe

(13) Ka : Ouiii

(14) Mé (à Kader) : c'est la lumière

(15) P : (qui montre la lampe et la remet derrière l'écran) elle est là la lampe, tu la vois plus.

(16) Ka:si

(17) P : non tu vois la lumière. Tu vois la lumière... Kader, t'es d'accord ou pas?

(18) Ka : Ouiii

(19) P : C'est ça la lampe, c'est ça que je veux pas que tu voies, là tu le vois pas!

(20) Ka: Oui, mais on voit le reflet un peu

(21) P : Tu vois le reflet?

(22) El : On voit la lumière

(23) Ka : La lumière

(24) P : Ce matin je te rappelle on a dit que la lampe éclairait dans toutes les directions, notamment les néons au-dessus de notre tête. (P. allume les néons), ces lampes là, elles éclairent partout.

(26) P : Il est bien évident que cette lampe là (P. montre la lampe utilisée dans l'expérience) elle éclaire partout

Ka : Oui

P: Moi ce qui m'intéresse c'est que tu ne puisses pas voir la lampe, la lampe directement d'accord?

P coupe court aux échanges avec Kader et enchaine alors avec la suite de la fiche pour la formalisation des observations et des interprétations.

Extrait 2 : classe de physique, min 18 et 19. Intervention de Kader, modifiant le milieu délimité précédemment par l'enseignante $(P=$ Professeur, $E l=$ Elèves non identifiés, $\mathrm{Ka}=\mathrm{Kader}$, Mé = Mélina) 
Lorsqu'il s'agit de vérifier que l'écran opaque positionné entre l'œil et la source empêche la vision de cette dernière, Kader conteste les faits (tdp 4, 6, 9) en s'appuyant sur la présence d'une lumière "visible * (lumière issue de la lampe et diffusée par les objets du voisinage). Si plusieurs interprétations peuvent être faites de cet épisode (par exemple : difficultés à séparer la « lumière » diffusée de la source primaire qui l'émet; ou encore, contestation d'un élève - que l'enseignante décrit comme "hyperactif ... agité ... ayant besoin $d u$ contact avec l'adulte pour chercher les limites pour tout le monde " (entretien ante) - plus destinée à « jouer » avec elle qu'à discuter le fond de l'affaire), c'est un autre point de vue qui nous intéresse davantage ici et sur lequel nous reviendrons immédiatement dans la conclusion de cette partie, celui prenant en compte la constitution du milieu. Nous avons souligné précédemment le soin avec lequel l'enseignante délimite le milieu, il reste que dans cet extrait 2, ce dernier est aussi tributaire de l'environnement matériel. On constate en effet que l'expérience réalisée interfère avec d'autres objets de la classe alors que ceux-ci ne font pas partie du milieu, au moins du point de vue de l'enseignante. Un phénomène semblable se produit au cours du second épisode (voir infra, extrait 6) où il s'agit de comprendre qu'un écran blanc peut, en diffusant la lumière d'une source primaire, éclairer un objet que celle-ci n'éclaire pas directement, et pour lequel la pénombre ambiante empêche d'observer le phénomène (extrait $6: \operatorname{tdp} 4,5$ et 10 , infra). On sait bien que l'étude en physique prend appui la plupart du temps sur un réel « reconstitué » pour s'affranchir des éléments non pertinents ou perturbateurs pour l'étude. Mais il arrive que malgré les précautions prises à cet effet par les enseignants, des éléments de l'environnement matériel «entrent » dans le milieu car perçus et explicités comme tel par un élève comme dans cet exemple. Enseignant et élèves doivent alors gérer la situation nouvelle. Ici (Extrait 2), le premier manipule les objets du milieu ( $\operatorname{tdp} 15)$ et amène d'autres éléments conceptuels (tdp 24 et 26), les seconds contribuent par leur prise de position (tdp 14 et 22) à renforcer l'identification des traits pertinents déjà faite par l'enseignante.

\section{Le milieu comme dimension problématique de l'action didactique conjointe}

Les deux épisodes d'EPS et de physique analysés rendent compte de phénomènes relativement récur- rents que nous avons pu observer dans les deux classes. Le milieu nécessaire à l'étude des savoirs relève d'un processus de co-construction qui n'est jamais totalement contrôlé ni garanti par le dispositif ou la tâche d'apprentissage. Comme l'ont mis en évidence de précédentes études (Comiti, Grenier, \& Margolinas, 1995; Mercier, 1997, 1998; PerrinGlorian \& Hersant, 2003; Rilhac, 2007; Roustan \& Amade-Escot, 2004; Thépaut \& Léziart, 2007), ce processus est problématique. S'il implique bien évidemment des aspects liés à l'épistémologie du professeur et aux conditions matérielles et symboliques structurant les dispositifs proposés aux élèves, au-delà, il est aussi le produit émergent de l'action conjointe professeur-élèves. Les deux illustrations du processus mésogénétique observé mettent en évidence la nécessité de certaines conditions - fortement liées aux spécificités disciplinaires et aux enjeux de savoir - pour que les tâches ${ }^{16}$ "fassent milieu ». Ainsi, si nous comparons les situations problématiques mises en place par les deux professeurs, nous pouvons dire que l'enseignant d'EPS ne peut pas compter sur le dispositif qu'il installe et manipule, pour délimiter un milieu effectif pour l'étude, contrairement à ce qui se passe en physique. Toutes les techniques mésogénétiques qu'il met en œuvre durant l'épisode décrit en prenant appui sur les réalisations des élèves (injonctions, démonstrations, régulations, invitations à réfléchir, etc.) ne peuvent aboutir car il ne peut pas prendre à sa charge ce qui est de la responsabilité des élèves : produire des frappes de dégagement suffisamment hautes pour obtenir les déplacements et replacements du joueur opposé tout en assurant la continuité des échanges. Il nous semble ici que la spécificité du savoir enseigné est une clé pour comprendre la situation. Contrairement au dispositif expérimental manipulé par l'enseignante de physique qui permet à celle-ci de prendre à sa charge, au moins au début de l'échange avec les élèves, la définition de ce (et seulement ce) qu'il sera convenu de débattre à propos des expériences observées, la tâche d'éducation physique ne permet au professeur (en tant qu'acteur isolé) d'installer les conditions initiales de l'étude. En effet, dans l'épisode de physique, la définition initiale d'un milieu didactique (délimiter ce qui doit être pris en charge par les élèves dans leur raisonnement) peut dans un premier temps relever de l'action isolée du professeur. On a vu cependant que la démarche d'investigation et de modélisation qui y est associée n'échappe pas aux difficultés de co- 
construction d'un milieu « générateur de possibles et de nécessaires » (Sensevy \& Quilio, 2002, p. 51), par exemple lorsque les élèves pointent des phénomènes apparaissant dans le milieu, liés à des objets considérés a priori comme extérieurs par l'enseignant. Du coté de la situation de badminton, l'enseignant échoue malgré toutes ses injonctions, à créer les conditions de la dévolution du jeu (Brousseau, 1986). Pour autant, l'analyse met en évidence, que ce dernier identifie pertinemment ce qui, dans les productions des élèves, pourrait enfin entrer en résonance avec son projet d'enseignement, ce qui lève le doute sur son épistémologie professionnelle ${ }^{17}$.

Envisageons maintenant la manière dont ces enseignants conduisent les interactions verbales en contexte difficile.

\section{Conduire en contexte difficile les interactions verbales avec les élèves : des manières de faire génériques}

Nous nous attachons dans cette deuxième section des résultats à examiner la façon dont ces deux enseignants conduisent les échanges avec leurs élèves. De nombreux travaux s'intéressent aux rôles joués par le(s) langage(s) dans la construction des connaissances en classe. Ils relèvent de différents cadres théoriques et convoquent des théories linguistiques diverses ${ }^{18}$. Pour ce qui nous concerne, nous envisagerons cette question à l'aune du triplet de genèses tel que proposé en théorie de l'action didactique conjointe en considérant avec Schubauer-Leoni et al. (2007) que la genèse du milieu est première dans le processus de construction de la référence (p. 58).

Une des caractéristiques fortes des deux enseignants observés réside dans l'intense activité déployée pour maintenir l'engagement de leurs élèves dans l'apprentissage : tous deux reconnaissent d'ailleurs qu'il faut "tenir la classe ", même si la gestion de la discipline ne fait pas l'objet d'un surinvestissement de leur part. Ils font en effet preuve d'une grande habileté à gérer les moments de désaffectation ou de décrochage des élèves. De nombreux exemples impossibles à développer dans le cadre de cet article - montrent que c'est dans la gestion concomitante de l'attention des élèves et du contenu de savoir que ces deux enseignants règlent l'avancement de la leçon (par exemple, voir le tour de parole 1 dans l'extrait 2 en physique, ou encore le tour de parole 5 de l'extrait 5 en éducation physique). On trouve ici la confirmation des tendances repérées par des recherches citées au début de cet article, selon lesquelles c'est dans une attention de tous les moments au bon fonctionnement de la classe, que peut être maintenue la relation didactique. Forts de ce constat, nous nous attachons ici à comparer l'action didactique de ces deux enseignants lorsqu'ils conduisent des échanges avec les élèves, tout en ayant en tête que les enjeux de ces échanges sont fondamentalement différents. Dans le cas de l'EPS, il s'agit de réfléchir aux conditions de l'action efficace pour dans un deuxième temps, retourner à la tâche afin d'en éprouver la réussite, alors qu'en physique, il s'agit de construire des assertions en relation avec le modèle de la vision par l'œil des objets non directement éclairés par des sources primaires et d'en institutionnaliser les éléments.

Dégager la règle d'action d'un dégagé efficace : un topos professoral en surplomb ne laissant quasiment aucun espace aux élèves

L'extrait retenu pour la comparaison est consécutif à la phase d'action des élèves dans la tâche de duo ci-dessus analysée. Il s'agit d'une phase d'échanges avec les élèves (min. 29 à 31 de la séance) lors de laquelle, l'enseignant ayant rassemblé les élèves face à lui au centre du gymnase, tente de poursuivre le processus de dévolution de la nécessité d'un dégagé haut permettant la continuité des échanges du volant (règle constitutive du jeu d'apprentissage proposé par l'enseignant, au sens de Sensevy, 2007). Cherchant à faire émerger la règle d'action rendant compte du placement sous le volant (posture et position du tamis par rapport au volant) et n'ayant pu l'obtenir dans la phase d'action précédente, il rassemble les élèves et conduit un épisode de questions - réponses dans lequel l'espace laissé aux élèves est minimal.

(1) P : alors dites le petit jeu là/ le petit jeu : il ne peut fonctionner il ne peut fonctionner que si vous tirez en hauteur donc on va repréciser certaines choses pourquoi parce que on s'aperçoit que certains d'entre vous ont du mal à tirer en hauteur pourquoi ils ont du mal à tirer en hauteur. Francky pourquoi vous avez du mal à tirer en hauteur? 
(2) Fr : parce que ce n'est pas assez fort

(3) $\quad \mathrm{P}$ : non ce n'est pas un problème de force

(4) Fr: parce que on ne tire pas

(5) El : non

(6) El : parce que il tire comme ça et il faut tirer comme ça (montre le geste de service et non le dégagé main haute)

$\mathrm{P}$ : non ça c'est le service, mais mais tu peux aussi trouver la solution sur ce geste-là alors? ... il faut prendre faut prendre la position pour tirer en hauteur et la position pour tirer en hauteur c'est laquelle? (brouhaha)

$\mathrm{P}$ : on s'aperçoit que certains jouent avec la raquette devant vous donc hein ils ont peur de ne pas voir la raquette et pas voir le volant / (...) regardez-là (face à toute la classe, démonstration d'une frappe de dégagement main haute) je mets ma raquette en arrière

$\mathrm{El}$ : faut prendre de l'élan

$\mathrm{P}:$ non

Ay : monsieur?

$\mathrm{P}$ : alors ...

Ay : pour frapper haut ...

P : donc ça veut dire et ... vous auriez dû le trouver de suite que pour frapper en hauteur il faut frapper le volant par-dessous (...) donc ça veut dire que en général en général vous ne vous placez pas assez sous le volant pour le frapper par-dessous pour le faire monter Il faut le faire monter donc il faut me le frapper en dessous

Extrait 3. Cours d'EPS, minutes 29 à 30 de la séance : un topos professoral envahissant

$(P=$ Professeur,$E l=$ Elèves non identifiés, $A y=$ Ayoub,

$$
\mathrm{Fr}=\text { Franky) }
$$

Dans cet échange, on perçoit une forme de conduite des interactions verbales où le professeur agit en surplomb, en invalidant les réponses des élèves qui ne sont pas conformes à celles attendues (celle de Francky et des autres élèves) et en résonant à la proposition d'Ayoub, ce qui le conduit très tôt (plus tôt que dans l'épisode parent en physique, voir infra) à refermer le débat. A partir de ce que nous a dit l'enseignant lors de l'entretien ante (" c'est assez dur parce qu'il faut franchir ce cap ... c'est-à-dire il faut arriver à ce que le collectif fonctionne »), nous faisons l'hypothèse que les risques de voir déraper la séance ainsi que la frustration de ne pas avoir obtenu des réponses satisfaisantes à la fois lors des mises en œuvre sur le terrain et lors du questionnement, amènent cet enseignant à réitérer les consignes et les démonstrations portant sur les traits pertinents («frapper par en dessous ») et non pertinents ("vous ne vous placez pas assez sous le volant ») de l'action à produire. Ce sont de notre point de vue autant de gestes professoraux participant à la construction d'une référence commune dans la classe (Schubauer-Leoni, 2008; Sensevy, Mercier, \& Schubauer-Leoni, 2000).

Mais au-delà, l'échange se poursuit, et l'extrait ciaprès, montre une accélération chronogénétique du fait de l'enseignant, afin de revenir à l'enjeu initial de sa séance (le déplacement de l'adversaire) dont on a vu qu'au fil de l'action, il avait du faire le deuil, et ce malgré l'insistance avec laquelle il nous avait présenté son projet de séance lors de l'entretien ante : "il faut les faire un peu sortir de l'échange pour ceux qui étaient encore dans l'échange sortir enfin l'exercice c'est un jeu continu qui les déplace qui ... qui comment on appelle ça qui développe le le leur vision du terrain de la largeur du terrain c'est-à-dire à travers un jeu continu il faut qu'ils essaient de jouer par exemple arriver à mettre l'adversaire dans un couloir aller le faire aller dans le couloir même si c'est du jeu continu ».

(1) P : Alors donc il faut frapper par-dessous ensuite qu'est-ce qu'il faut faire

(2) El : il faut ..

(3) Un autre El : il faut regarder le volant

(4) P : regarder le volant oui et puis puis et puis ... après ... continue comme ça ... dans cette voie

(5) P : NATACHA ! je ne te dérange pas quand je fais cours (intervention sur le comportement)

(6) Kh : monsieur, monsieur il faut se placer

(7) $\quad \mathrm{P}$ : se placer et après ...quand on regarde la position de l'adversaire! ... mais qu'est-ce que tu fais quand tu regardes l'adversaire

(8) El : on regarde le volant

(9) P : le volant et l'ad... ça veut dire quoi (...) si je vous dis qu'il faut regarder l'adversaire et le terrain adverse ça veut dire que pendant tout ce que vous faites il faut toujours être face au filet ou dans la direction du terrain adverse / hein /donc quand vous vous déplacez pour jouer/ quand vous vous déplacez pour jouer / vous devez penser à : pour pouvoir jouer il faut que je sois face au filet parce que je vois l'adversaire et il faut jouer là où il n'est pas etc. etc. donc ça veut dire qu'il faut se débrouiller de se déplacer 
sans perdre de vue le terrain adverse d'accord Alors maintenant, toujours sur cet exercice, on va faire un petit jeu...

Extrait 4, minute 31 de la séance d'EPS : retour sur les enjeux initiaux

$(P=$ Professeur,$E l=$ Elèves non identifiés, $K h=$ Khaled $)$

Cet extrait montre que, bien que le milieu de l'action n'ait pas permis d'engager les élèves dans le travail du déplacement de l'adversaire pour créer une situation favorable à l'attaque de la cible, l'enseignant ne perd pas de vue son intention initiale de prise d'information sur le terrain adverse. Rappelons qu'il s'agit d'un enjeu tactique essentiel donnant sens à la réalisation d'un coup de dégagement en badminton. C'est sur cette assertion professorale que se clôture l'épisode, l'enseignant renvoyant les élèves au milieu de l'action, c'est-à-dire à la tâche décrite lors du premier épisode, car en EPS (comme sans doute dans toutes les disciplines), l'institutionnalisation et la validation du savoir ne peuvent se passer de la réussite en acte (Amade-Escot, 2005).

D'une démarche maïeutique à une position en surplomb en physique

L'enseignante de physique cherche, à partir d'une démarche maileutique, à faire produire aux élèves la réponse aux questions qu'elle pose comme on peut le voir, par exemple, dans la phase de réflexion précédant l'expérimentation rapportée dans l'extrait 1 (tdp 3 à 12 , puis 13 à 25). Cette démarche est habituelle chez elle, mais on voit au fur et à mesure que cette séance en classe entière ${ }^{19}$ avance, qu'elle rencontre des difficultés de plus en plus importantes. La première apparaît dans le tour de parole 26 de l'extrait 1. L'enseignante finit par prendre à sa charge le raisonnement selon lequel il est impossible de voir une source lumineuse si on place un écran opaque entre l'œil et cette dernière, raisonnement que les élèves ont du mal à terminer. On pourrait penser que cette situation est conjoncturelle, liée au fait qu'elle fasse raisonner les élèves tout au long de cet épisode sur la non-visibilité de la source primaire pour qu'ils en déduisent in fine et a contrario les conditions nécessaires à sa vision, ce qui pourrait être considéré comme une difficulté. Mais, un peu plus tard dans la séance (cf. extrait 5 ci-après), on peut percevoir un comportement de même type. L'enseignante demande d'expliquer, au cours d'une discussion précédant l'expérimentation pourquoi on la voit (elle) dans la classe alors qu'elle n'est pas directement éclairée par une source primaire de lumière.

(1) P : La lumière, elle arrive du soleil, jusque là... SAMY! La lumière arrive du soleil. (Samy et Kader ne tiennent pas en place mais ne semblent pas encore à côté de la tâche)

(2) Mé : Oui

(3) P : Ensuite?

(4) Mé : Elle passe par la fenêtre

(5) $\quad \mathrm{P}$ : elle passe par la fenêtre... Après?

(6) Mé : Elle se diffuse dans la pièce

(7) $\quad \mathrm{P}$ : Elle se diffuse dans la pièce Explique moi ça

(8) Mé : bé...

(9) Si : C'est comme l'air...

(10) Mé : elle occupe la pièce

(11) Mé : c'est comme l'air...

(12) Mé : Ça occupe toute la pièce

(13) P : Comment ça se fait qu'elle va dans toute la pièce?

(14) Mé : ben parce que...//

(15) Mo : elle réfléchit

(16) P : elle réfléchit?

(17) Mé : elle se diffuse

(18) P : Ah elle est diffusée effectivement //

(19) Sa : partout dans la pièce

(20) P: par les objets qui nous entourent (respiration). Elle est diffusée par les objets qui nous entourent

Extrait 5 : classe entière de physique, minute 38. Surexploitation des propos de Mélina relatifs à la diffusion de la lumière par les objets de l'environnement.

$(P=$ Professeur, Mé = Mélina, Mo = Moktar, Si = Sihène, Sa = Samy)

Dans cet extrait, Mélina et Sihène tiennent des propos (tdp 3, 6, 7, 8, 9 et 14) qui peuvent être rapprochés de la conception du «bain de lumière » identifiée par les travaux cités précédemment. Les élèves ne parvenant pas à formaliser l'interprétation attendue, l'enseignante "sur-exploite » l'affirmation de Mélina pour parvenir à la conclusion qu'elle peine à instaurer (indice d'un effet Jourdain). Elle transforme la réponse de cette élève : "elle (la lumière) se diffuse " (tdp 17) en une assertion plus adaptée à son projet : « ah, elle est diffusée effectivement » (tdp 15). Elle pour- 
suit en rebondissant sur le complément apporté par Samy (tdp 19: "partout dans la pièce " qu'elle reformule en "par les objets qui nous entourent » (tdp 20), ce qui lui permet alors d'énoncer la conclusion qu'elle attendait des élèves «elle est diffusée par les objets qui nous entourent " $(\operatorname{tdp} 20)$ à laquelle elle espère donner un statut de référence dans la classe.

L'analyse que l'on vient de faire des extraits 1 et 5 montre ponctuellement une prise en charge partielle par l'enseignante du raisonnement qu'elle souhaite faire élaborer par les élèves. Ce processus topogénétique dans lequel, jusqu'aux deux tiers de la séance, les élèves ont encore une place, se radicalise à la fin de celle-ci, comme on peut voir dans l'extrait 6 ci-après. Celuici rapporte les interactions à propos de l'expérience réalisée pour répondre à la question « pourquoi voit-on les objets autour de nous alors que souvent ils ne sont pas directement éclairés par une source primaire? " (Fiche élève). Les interactions visent à amener les élèves à interpréter les observations réalisées (cf. Figure 1) : dans la salle qui devrait être plongée dans l'obscurité complète, une balle rouge, non éclairée directement par une lampe en raison de la présence d'un objet opaque entre celle-ci et la balle, est rendue visible par la présence à proximité d'une feuille blanche. Ce phénomène (que l'éclairage ambiant rend moins perceptible) peut être interprété comme résultant de la diffusion par un écran blanc de la lumière émise par la lampe.

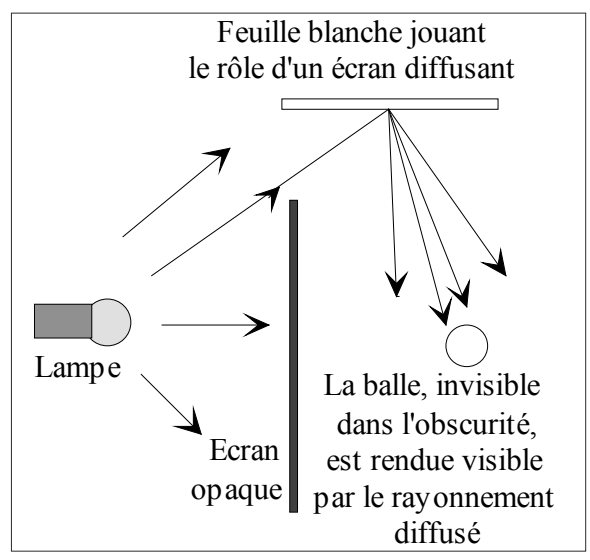

(1) P: Donc je maintiens ma lampe allumée; d'accord? j'ai mon objet coloré; l'objet coloré, je vais le mettre sur le côté. Lobjet coloré (Chuchotements, discussions à voix basse) l'objet coloré, vous l'apercevez, éclairé en lumière blanche il est...
(2) Ho : Rouge!

(3) P : c'est un objet rouge, il y a pas de souci.... Si je décide... si je suis dans le noir, bien évidemment ce n'est pas le cas, si je décide de ne plus laisser voir cet objet (P place un écran entre la lampe et la balle rouge), il faut pour cela

(4) Ka: On le voit quand même

(5) P sur le même ton: je te dis Kader, imagine que l'on soit dans le noir complet!

(6) Ka : oui

(7) $\quad \mathrm{P}$ : Donc là, quoi qu'il en soit Kader, la lampe n'éclaire pas mon objet. On est d'accord ou pas?

(8) Une fille : oui

(9) P : Qu'est ce qu'il se passe avec mon écran blanc là? (l'écran blanc diffuse la lumière qu'il reçoit et éclaire la balle, le tout étant difficile à percevoir en raison de la lumière ambiante).

(10) Ka : Rien

(11) El : Y renvoie

(12) El : Il donne la lumière

(13) P : C'est une source primaire ou secondaire?

(14) Elèves : (les uns) Primaire! - (les autres) Secondaire!

(15) P : SECONDAIRE! Alors rappelez moi comment fonctionne une source secondaire!

(16) Mo : elle reçoit de la lumière

(17) P : Elle reçoit de la lumière et elle en diffuse...

(18) El : elle diffuuse!

(19) Ka : elle émet!

(20) P : elle en diffuse une partie! Et donc si je place l'objet dans la lumière que diffuse mon écran blanc, Qu'est ce qui va se passer?

(21) El : On voit de la lumière rouge

(22) P : On voit pas de lumière rouge, c'est l'objet qui est rouge...

(23) El : Elle [la balle] va pas devenir rouge!

(24) P : Bon on est d'accord? Je peux arriver à récupérer la lumière de la lampe alors que vous ne voyez pas directement la lampe et j'ai utilisé mon écran blanc comme une source secondaire qui va me permettre d'éclairer mon objet.

Extrait 6, cours de physique minutes 40 à 41 : prise en charge par l'enseignante de la construction de la référence $(P=$ Professeur, Ho = Housseme, Ka = Kader, Mo = Moktar ; $\mathrm{El}=$ élève non identifié)

Pour faire aboutir les interprétations, l'enseignante ferme considérablement les questions posées et rectifie elle-même les réponses lorsque celles-ci ne correspondent pas à celles qu'elle attend (tdp 15, 17, 
20, 22, 24). Comme l'enseignant d'EPS, elle adopte ainsi à ce moment-là un topos en « surplomb». En lien avec cette position comme on peut le voir, les élèves participent a minima (tdp 10, 11, 12, 14, 18, 19) voire plus du tout. Un peu plus tard en effet, elle est contrainte d'avancer seule, attendant intervention après intervention une réponse des élèves qui ne vient plus (min. 43) :

$P$ : on vient de voir que notre écran blanc, diffuse la lumière, donc il renvoie ... Amelle! ... il renvoie ... Housseme assis, je t'assure que tu seras beaucoup mieux ... il renvoie ... L'écran blanc renvoie la lumière ... Issue de la source!

L'enseignante prend ici totalement à sa charge la construction de la référence avec des élèves qui échangent peu entre eux et font souvent des réponses minimales. Les réductions successives qu'elle opère à partir des réponses des élèves jugées insuffisantes, conduisent à fermer progressivement le milieu du débat d'une façon relativement identique à celle de l'enseignant d'EPS, même si les formes linguistiques de cet épisode semblent moins monologales que les précédentes.

Les premiers tours de parole laissent présumer des raisons plausibles à ce comportement : i) la nature de l'environnement matériel est peu propice à l'observation du phénomène à interpréter, celle-ci requérant l'obscurité complète alors que la classe est dans une pénombre très lumineuse ( $\operatorname{tdp} 4,5,7$ et 10$)$; ii) l'attention et l'implication consenties par les élèves en cette fin de séance réalisée en début d'après-midi est très faible (tdp 1, 14, 18). Mais les difficultés de l'enseignante apparaissent surtout au moment où elle tente de faire passer les élèves d'un discours que l'on peut relier au « genre primaire », « qui prend forme dans une communication non médiée, qui est en relation immédiate avec la réalité concrète et les propos des autres » à un discours de " genre secondaire », qui «se développe au sein d'une communication culturellement plus complexe, et comparativement plus développée et organisée », et qui aboutit donc à un ensemble plus formalisé auquel appartient notamment le discours scientifique (Bakhtine, 1986, p. 62). L'extrait 1 précédent met en évidence, aux tours de parole 19 à 26, de semblables difficultés lorsque les élèves argumentent en parlant du "truc opaque " (l'écran), " pas transparent » et qui « renvoie » (la lumière), alors que l'enseignante essaie de leur faire formuler qu'il est impossible de voir la source lumineuse s'il existe un écran opaque sur le trajet de la lumière entre la source et l'œil. On le voit encore dans l'extrait 5 quand les élèves interprètent la vision d'objets non éclairés directement pas une source primaire en avançant que la lumière "se diffuse dans la pièce ", que "c'est comme l'air", "qu'elle occupe la pièce " alors que l'enseignante tente de leur faire formuler que la lumière du soleil est diffusée par les objets de la pièce dans toutes les directions.

Les interactions verbales comme descripteurs des dynamiques mésogénétiques

En conclusion, l'analyse comparative des interactions verbales dans les extraits précédents fait apparaître au fil des échanges, à la fois en EPS et en sciences physiques, la place de plus en plus importante occupée par les enseignants en lien avec le désengagement progressif des élèves. Cette dynamique du partage topogénétique aboutit à une position enseignante en surplomb dans la construction de la référence. Elle a pour conséquence d'actualiser un milieu peu propice à la dévolution. Nous faisons l'hypothèse que les deux enseignants, malgré leur désir de mettre en œuvre des moments de formulation collective sont amenés au fil de leur séance à contrôler les prises de parole des élèves, pour éviter toute digression dont chacun d'eux a pu dire lors des entretiens qu'elles constituaient des risques de dérapage : " moi j’ai du mal à laisser faire parce que après parce que si après on laisse faire je pense que ça peut aller très loin dans le désordre " (enseignant d'EPS, entretien post); « à partir du moment où ils sont en classe entière, c'est pas que sur cette classe, c'est sur l'ensemble de mes classes, je suis beaucoup plus obligée de prendre la classe en main et d'être dirigiste. Si je laisse s'instaurer les mêmes débats que ce que j'ai fait ce matin [en demi classe], très rapidement je ne tiens plus la classe »(enseignante de physique, entretien post). Ce phénomène, en partie générique car largement mis en évidence tant au niveau des recherches didactiques que dans des études socio-linguistiques sur le questionnement en classe (Carlsen, 1991; Lobato, Clark $\&$ Burns Ellis, 2005), nous semble devoir être aussi analysé en termes de mésogenèse.

Les dynamiques observées confirment enfin que l'analyse des interactions verbales ne peut être coupée 
d'une prise en compte spécifique, épistémique, des enjeux de savoirs qu'elles portent : en EPS, faire advenir lors des échanges la nécessité d'une frappe vers le haut, est un raisonnement préalable à l'action et donc à l'apprentissage visé qui ne préjuge en rien de son succès; de la même manière en physique, l'assertion conclusive relève de l'aboutissement de l'étude, même si rien ne garantit l'appropriation effective par les élèves du modèle proposé. Sous des formes différentes, nous pouvons dire que dans ces deux disciplines, le contexte difficile de l'enseignement observé exacerbe, lors des phases de mises en commun, les risques d'un affaiblissement des savoirs étudiés, même lorsque les enseignants ont des intentions didactiques fortes. Ce constat explique en partie les stratégies maïeutiques qu'ils mettent en œuvre, d'autant plus que le nombre d'élèves dans la classe (15 et 17) permet peu ou prou de tous les associer dans la tâche, ce qui, aux dires des enseignants, est encore plus nécessaire dans ces contextes qu'ailleurs.

\section{Une difficile dynamique mésogénétique en contexte difficile}

Comme ils l'ont annoncé dans les entretiens préalables, ces deux enseignants tentent de faire vivre aux élèves des expériences d'éducation physique et de sciences physiques ayant une certaine épaisseur épistémique. Ils ne se contentent pas en effet de proposer quelques micro-tâches très individualisées permettant d'occuper tous les élèves en favorisant leur réussite immédiate comme décrit par les recherches citées en début d'article. Au prix d'un investissement de tous les instants, les pratiques de ces deux enseignants sont à rapprocher du troisième i-genre ${ }^{20}$ décrit dans l'ouvrage dirigé par Peltier-Barbier (2004). Les professeurs appartenant à cet i-genre proposent des scénarios d'enseignement et d'apprentissage prenant en compte les contenus enseignés à partir de «situations problèmes complexes en laissant aux élèves du temps dans leur recherche, sans trop de négociation à la baisse » (p. 78) et instaurent des moments de synthèse collective fortement étayés (ibid, p. 100-101). Toutefois, l'analyse comparée de l'action conjointe professeur-élèves en EPS et en physique, met en évidence, malgré les tentatives de mise en œuvre de scénarios didactiques ambitieux, que la construction d'une référence conjointe semble toujours plus hasardeuse et difficile à faire aboutir au fur et à mesure que la séance avance. Nous avons observé que cette logique fonctionne si les élèves sont partenaires de l'action de l'enseignant, s'ils sont engagés dans les taches proposées. On a pu voir que sous certaines conditions (identifier clairement ce qu'est l'enjeu de savoir, intervenir pour que la tâche proposée aux élèves puisse «faire milieu », se centrer sur l'essentiel et le souligner fortement, amener la conclusion pour couper court à des échanges improductifs, privilégier l'action en EPS et l'oral en physique), l'engagement disciplinaire productif (Engle \& Conant, 2002; Herrenkoh \& Guerra, 1998) des élèves était possible au moins en début de séance. Néanmoins, la pression temporelle et les modes de participation hétérogènes des élèves, les difficultés de faire vivre les dispositifs initiaux, les obstacles rencontrés par les élèves dans la production d'un discours « secondaire " sur l'action ou l'observation ont pour conséquence en EPS comme en physique (et selon des spécificités que nous avons tenté d'éclairer) des difficultés à maintenir un milieu heuristique pour l'étude. Ainsi, dans ces deux séances, la communication est toujours de type « authoritative ${ }^{21}$ " (Mortimer $\&$ Scott, 2003, p. 33) l'enseignant se positionnant uniquement d'un seul point de vue, celui que font valoir l'EPS ou la physique, et elle devient de moins en moins interactive.

Malgré des interventions réitérées et systématiques, les enseignants n'arrivent pas toujours à générer avec leurs élèves les conditions d'un milieu didactique approprié relativement au savoir visé ${ }^{22}$. Ils prennent tous les deux - quoique de façon fort spécifique - en charge la production de la référence, se positionnant du coup « en surplomb» (Schubauer-Leoni, 2008) pour faire avancer le savoir. Cette stratégie s'accompagne d'un ensemble de techniques visant dans un même temps à gérer l'ordre dans la classe et à rendre les élèves partenaires de la construction de la référence sans pour autant réellement y parvenir ${ }^{23}$. Dans ce cas, les élèves participent peu aux échanges et les enseignants apportent beaucoup d'informations, la chronogenèse s'accélère. Ce processus est vraisemblablement spiralaire : l'élève désertant partiellement l'espace didactique, l'enseignant est alors contraint de l'occuper pour maintenir la relation didactique, et ce faisant, il amène l'élève à le déserter un peu plus. Une des conséquences est qu'une partie de l'activité didactique se trouve empêchée tant au niveau des élèves que des enseignants. On voit là combien 
sont fragiles les équilibres en contexte difficile. En même temps, ces phénomènes ici exacerbés, ne sont pas tant étrangers que cela à ceux que chacun des auteurs a pu observer en EPS et en physique dans des établissements plus ordinaires voire favorisés (Amade-Escot, 2003; Venturini, Calmettes, AmadeEscot, \& Terrisse, 2007).

Il reste que cette étude en mettant en exergue les difficultés récurrentes de deux enseignants à rendre effectifs des milieux didactiques adéquats aux enjeux de savoirs visés, pose la question théorique centrale à notre avis, des conditions de possibilités et de fonctionnement des milieux didactiques. Ceci nous amène dans la dernière partie de cet article à revenir sur les concepts de milieu et de mésogenèse et leur statut dans la théorie de l'action didactique conjointe.

\section{Milieu et mésogenèse : contribution théorique à l'approche comparatiste en didactique.}

\section{Le constat d'une "fragilité ${ }^{24} " d u$ milieu en contexte difficile}

Les extraits que nous venons d'analyser relatifs aux séances d'EPS et de physique en collège « ambition réussite » confirment les études menées dans d'autres disciplines et mettent l'accent sur les façons dont les enseignants tentent de les résoudre tout en pointant les difficultés et les contradictions auxquelles ces derniers sont confrontés. L'apport de cette étude est de montrer que malgré leur conviction et leur implication pour faire vivre aux élèves des séquences d'apprentissage denses en savoirs, on assiste, au fil des interactions didactiques à une altération progressive des milieux didactiques en liaison avec les degrés d'engagement des élèves dans le processus conjoint. Nous ne concluons pas pour autant sur l'affaiblissement épistémologique inéluctable du processus d'enseignement en contexte difficile. Au contraire, nos analyses ont mis en évidence tant au niveau de la séance, que sur la temporalité plus longue de plusieurs séances (Debars, 2005; Venturini \& Amade-Escot, soumis) que des apprentissages sont possibles. L'objectif de cette section est autre. Nous souhaitons, dans une perspective d'approfondissement théorique, revenir sur les concepts de milieu et de mésogenèse à l'aune des observations effectuées.
L'analyse des corpus empiriques a mis en avant le constat d'une certaine instabilité des milieux initiaux selon des configurations mettant en jeu à la fois : i) la spécificité du savoir enseigné (par exemples dans notre étude, le savoir relatif à la frappe de dégagement, ou à la diffusion parasitée par la pénombre trop lumineuse pour qu'elle puisse être observée); ii) le type de régulation ou d'étayage accompli par le professeur (dont rendent compte les extraits rapportés); iii) l'engagement (ou non) des élèves dans le travail demandé. Ainsi, l'enchâssement du jeu du professeur sur le jeu de/s élève/s contribue à transformer le milieu initial selon diverses modalités : par exemple en délimitant ce qui doit être pris en charge par les élèves ou en produisant, par des effets topogénétiques en surplomb, l'avancée du savoir pour ne citer que quelques effets précédemment rapportés. Un second constat, perceptible à la lecture des extraits précédents met en évidence combien ce processus conjoint s'appuie sur et produit des phénomènes différentiels selon les élèves (SchubauerLeoni, 1996), par exemple : en EPS, Ayoub (extrait 3 , tdp. 11 à 14) et Soraya (cf. premier épisode); en physique, Mélina et Kader (extrait 2, tdp. 4 à 14). L'analyse nous permet de conclure avec d'autres, que le « milieu de l'enseignant » ne recoupe que partiellement celui de chacun des élèves ce qui a des conséquences sur leurs trajectoires ou leurs biographies didactiques (Comiti, Grenier, \& Margolinas, 1995; Mercier, 1997, 1998; Perrin-Glorian \& Hersant, 2003; Rilhac, 2007; Thépaut \& Léziart, 2007). Quel statut alors donner au concept de milieu dans la théorisation de l'action didactique conjointe? Avant d'entamer cette discussion, il nous semble opportun de rappeler quelques éléments fondateurs relatifs à cette notion didactique.

\section{À l'origine deux visions, antagoniste ou institu- tionnelle, du milieu didactique}

On doit à Brousseau $(1986,1990)$ la mise en évidence de la nécessité d'un milieu, lui-même finalisé par rapport aux connaissances mathématiques visées. Comme l'indiquent Perrin-Glorian (1999) et Salin (2002) cette notion, centrale en théorie des situations didactiques, a été précisée plus tardivement. Initiée à partir de la conception piagétienne, tout en s'en distinguant notablement comme l'indique la citation qui suit, la modélisation du milieu dans la théorie 
des situations didactiques se réfère explicitement à l'apprentissage par adaptation (Orange, 2007 ; PerrinGlorian, 1994; Salin, 2002).

« L'élève apprend en s'adaptant à un milieu (...) Ce processus psycho-génétique piagétien est à l'opposé du dogmatisme scolastique. L'un ne semble rien devoir à l'intention didactique, alors que l'autre lui doit tout. En attribuant à l'apprentissage naturel, ce qui repose sur l'art d'enseigner selon le dogmatisme, la théorie de Piaget risque de soulager le maître de toute responsabilité didactique: ceci constitue un paradoxal retour à une sorte d'empirisme! Mais un milieu sans intention didactique est manifestement insuffisant à induire chez l'élève toutes les connaissances culturelles que l'on souhaite qu'il acquière » (Brousseau, 1986, p. 49).

L'exigence, fondamentale chez Brousseau, d'un « milieu adidactique, antagoniste de l'élève " est résumée par Salin (2002) autour de trois idées princeps : «i) le milieu doit être facteur de contradictions, de difficultés, de déséquilibres donc d'adaptation pour l'élève; ii) le milieu doit permettre le fonctionnement "autonome » de l'élève; et iii) l'apprentissage doit conduire à la maîtrise de savoirs mathématiques identifiés comme tels, et pas seulement de connaissances» (p. 114). Néanmoins, la question du milieu adidactique sera par la suite beaucoup discutée, notamment lorsque les travaux, quittant en partie le paradigme de l'ingénierie didactique, se centrent sur l'analyse de séances ordinaires (Perrin-Glorian, 1999). La discussion affrontera alors le problème de l'existence même d'un milieu « adidactique ». L'évolution du focus des recherches a eu d'abord pour conséquence un développement du modèle en termes de «structuration du milieu » (Brousseau, 1990; Margolinas, 1995, 1999) mettant l'accent sur l'emboîtement des différents niveaux de lecture que peut faire le chercheur des actions du professeur et des élèves. Puis des extensions de la notion sont apparues identifiables par les qualificatifs associés : milieu matériel, objectif, allié, potentiel, effectif, etc. (pour un développement voir Salin, 2002). L'importation du concept dans d'autres didactiques contribuera aussi à questionner la modélisation « antagoniste » du milieu. Retenons pour le moment l'idée d'un milieu adidactique « d'abord associé à une situation locale, 'située' mais examinée d'un point de vue global »(Salin, 2002, p. 120). Rappelons en effet, que ce concept articule deux échelles différentes : « une échelle un peu globale où il s'agit de déterminer un milieu pour l'apprentissage d'un savoir, à laquelle se rattache l'idée de situation fondamentale, et une échelle plus locale d'étude d'une situation à laquelle se rattache la structuration du milieu » (Perrin-Glorian, 1999, p 285). Enfin, pointons pour la discussion qui va suivre, même si nous laisserons momentanément sous-jacente cette question, que le milieu est indissociablement lié au contrat didactique comme l'indique le titre de l'article éponyme de Brousseau (1990) car ces deux notions sont aux principes même des « transactions didactiques » (Sensevy, 2007).

Mais traiter du « milieu » en didactique des mathématiques impose de considérer aussi l'approche qui en est faite en théorie anthropologique du didactique (Chevallard, 1992). Pour cet auteur, qui se place du " point de vue de l'écologie des systèmes didactiques » (p. 103) le milieu est une des conditions nécessaires (avec le contrat) à leur fonctionnement. Il faut qu'à chaque instant un ensemble d'objets puisse exister pour les sujets de l'institution, à savoir qu'il existe a minima des « rapports institutionnels localement stables». Dans l'article cité, qui postule l'articulation des deux théories didactiques (articulation qui sera reprise par Perrin-Glorian, 1999), Chevallard se focalise sur les conditions de possibilité du fonctionnement didactique (quelle qu'en soit la pertinence, que ce fonctionnement soit « bon ou moins bon ») ce qui présuppose l'existence d'un milieu, dit institutionnel, sorte de préalable à tout projet d'enseignement. Dans ce cadre, l'auteur indique que le fonctionnement d'un système didactique fait « bouger » le milieu puisque les rapports personnels aux objets d'enseignement vont se transformer au fil du temps didactique. Est introduit ici le deuxième terme mis en discussion dans cet article, celui de mésogenèse (ou genèse du milieu) : «à chaque instant, le milieu apparaît subjectivement comme un donné; mais c'est en vérité un construit permanent » (Chevallard, 1992, p. 95). L'évolution récente de la théorie anthropologique du didactique reconfigure cette question autour de la « dialectique des media et des milieux » (Chevallard, 2007) mettant l'accent sur les fonctions médiatrices jouées par le milieu (et ses ingrédients) dans l'étude des savoirs sans pour cela nécessairement recourir à une modélisation " antagoniste » du concept et permettant d'intégrer à l'analyse les formes plus expositives d'enseignement. 


\section{Un concept travaillé et enrichi par son usage dans différentes didactiques}

Le concept de milieu a fait l'objet d'un assez grand nombre de travaux en didactique des mathématiques et a été discuté à l'occasion de son importation dans d'autres didactiques ${ }^{25}$. Il en ressort quelques constats récurrents et la mise en discussion de sa valence « antagoniste ».

\section{Des points de convergences}

- Une première convergence réside dans le fait que le milieu didactique résulte d'un processus évolutif qui s'appuie sur des aspects institutionnels en liens avec des usages, des habitudes, des formes de travail plus ou moins légitimés. Le milieu de la théorie anthropologique est défini « en référence à une institution et une temporalité institutionnelle » (Perrin-Glorian, 1999, p. 288). Ce point de vue est décisif selon l'auteure pour l'analyse de séquences dans des classes ordinaires. Mais il rend compte aussi d'une propriété bien mise en évidence par la notion de mesogenèse introduite par Chevallard (1992) et reprise par les didacticiens comparatistes, selon des perspectives que nous aborderons plus loin (Johsua \& Félix, 2002; Sensevy, Mercier, \& SchubauerLeoni, 2000; Sensevy \& Mercier, 2007)

- À ces aspects institutionnels s'ajoute l'idée partagée que le processus mésogénétique relève d'une action conjointe du professeur et des élèves ce qui suppose de l'étudier à l'échelle locale selon « les conditions et les contraintes spécifiques d'une situation didactique dans sa dimension praxéologique $»($ Thevenaz-Christen, 2002, p. 56).

- Enfin, les auteurs s'accordent pour considérer l'émergence au fil des interactions didactiques de plusieurs milieux en raison même des dynamiques qui soutiennent le processus mésogénétique. Comiti, Grenier, \& Margolinas (1995) ont mis en évidence des bifurcations possibles du milieu en lien avec le « dédoublement des situations » qui rend compte du fait que le milieu didactique dans lequel évolue un certain nombre d'élèves n'est pas celui supposé par le professeur. Un feuilletage apparaît ainsi, permettant de saisir - à partir du modèle de structuration du milieu initié par Brousseau (1990) et poursuivi par Margolinas (1995, 1999) -que certains objets au lieu d'appartenir au milieu objectif deviennent eux-mêmes des enjeux d'apprentissage (comme par exemple dans notre étude, l'objet frappe de dégagement en badminton, dont on peut penser que l'enseignant considère qu'il est acquis, et qui devient en fait l'enjeu de toutes ses régulations lorsqu'il passe de groupe en groupe). C'est en raison de ces bifurcations que le professeur adopte certains types de gestion, comme celui de s'appuyer sur le bon élève (par exemple en physique, Mélina, dans l'extrait 2), celui qui semble fonctionner dans le «bon milieu » (Comiti, Grenier, \& Margolinas, 1995, p. 110). D'une manière générale, le développement des études en classes ordinaires fait émerger le pluriel de la notion. Les distinctions entre "milieu du professeur » et " milieu de/des élève/s », celle de " milieu potentiel » et de « milieu activé » (ou encore « effectif » : ce qui dans l'environnement de l'élève lui apporte des rétroactions, Perrin-Glorian, 1999) en rendent compte. Ces distinctions, toutes en filiation avec la théorie des situations de Brousseau, maintiennent l'idée capitale d'un milieu « adidactique ».

Une valence " antagoniste » sujette à discussion

Au-delà des convergences observées, il apparaît toutefois que la valence « antagoniste " du milieu semble quant à elle plus problématique. Ce point fondamental dans la modélisation de Brousseau ${ }^{26}$ est en effet, discuté par différentes didactiques lorsqu'elles importent ce concept pour leurs usages :

- En didactique de l'EPS, les chercheurs pointent les réductions possibles, voire la naturalisation de la notion, sous forme d'un retour à une forme d'empirisme naif en rabattant le concept sur les dimensions matérielles de la tâche prescrite ${ }^{27}$. Quelques recherches ont conclu sur la difficulté, voire l'impossibilité, au fil des interactions de maintenir à proprement parler un milieu antagoniste de l'élève (Rilhac, 2007; Roustan \& Amade-Escot, 2004). En EPS, les rétroactions du milieu (au moins certaines d'entre elles) sont difficilement modélisables a priori car elles ne peuvent être pensées indépendamment du type d'actions motrices produites (ou qui seront produites) par les élèves, elles-mêmes constitutives de l'agencement du milieu, comme dans les extraits en badminton rapportés dans cet article. Ainsi, s'il existe bien en EPS 
- comme dans les autres disciplines - des nécessités du milieu pour que l'élève fasse l'expérience cruciale d'un rapport au savoir en référence à des pratiques sociohistoriquement cristallisées en activités (SchubauerLeoni et al. 2007, p. 53), il reste que l'expérience de ces nécessités est indexée aux formes de pratiques mettant en jeu le savoir. Cette spécificité rend plus ardue l'identification et le contrôle des variables de commande des dispositifs initiaux et leur caractère antagoniste (Amade-Escot, 2005).

- Au fil des migrations du concept d'une didactique à une autre, les questionnements liés aux spécificités des enjeux de savoirs conduisent certains chercheurs à récuser l'idée même d'un milieu antagoniste. En physique, Johsua (1996a) souligne la difficulté à penser un milieu antagoniste en raison de l'impossibilité d'y inclure tous les éléments pertinents et seulement ceux-là ${ }^{28}$. Tsoumpélis (2005) développe pour sa part l'idée que la composante matérielle du milieu (sur laquelle s'appuient l'observation, la manipulation et l'expérimentation en lien avec des prédictions) appartient au monde réel, et de ce fait est « externe aux sciences physiques ${ }^{29}{ }$. C'est pourquoi, pour cet auteur, « il n'y a pas de véritables rétroactions du milieu concernant les explications du phénomène. Les rétroactions ne seraient pertinentes que pour l'aspect mathématique du processus. Une infinité d'explications peuvent être associées, en théorie au formalisme mathématique qui prédit un aspect du processus en question ». Aussi, la validation par le recours à l'expérimental est complexe, « une réponse négative pouvant porter sur soit l'aspect explicatif, soit sur l'aspect mathématique qui lui est associé soit sur les deux », ce qui n'est pas selon l'auteur, sans poser « a priori des limitations sévères sur les perspectives de la situation adidactique » (p. 217) Ainsi, dans notre corpus, en est-il, par exemple, des explications selon lesquelles le caractère opaque de l'écran serait du à l'impossibilité pour la lumière de le traverser (propos de l'enseignante, extrait 1 , tdp 26), ou encore à la réflexion de la lumière (propos d'un élève, extrait 1 , tdp 23 et 25). Le milieu matériel convoqué ne peut fournir seul les rétroactions permettant à l'élève d'avancer vers le savoir en jeu.

- D'une certaine manière, nous retrouvons ici l'argumentaire développé par Orange (2007) à propos «l'apodicticité » des savoirs des sciences de la vie et de la terre (p. 38) qui le conduit à ajouter à la structuration verticale du milieu didactique telle que remaniée par Margolinas (1995), une structuration horizontale sous forme d'un triptyque inspiré par les travaux du physiologiste Claude Bernard relativement au concept de «milieu intérieur ${ }^{30} »$. S'appuyant sur l'analyse d'une séance portant sur le mouvement du membre supérieur chez l'homme, il propose de distinguer le milieu didactique intérieur de la classe, le milieu didactique extérieur à la classe, et le milieu didactique intérieur de l'élève (Orange, 2007, p. 48 à 53). Selon Orange, le milieu objectif (extérieur à la classe) ne peut seul assurer toutes les rétroactions nécessaires pour la validation des modélisations que l'élève produit. Celles-ci doivent en effet obéir à des contraintes (des nécessités) que l'élève construit au cours de son activité de problématisation, avec l'aide de l'enseignant et des documents qu'il apporte. Ainsi, le milieu intérieur comporte pour l'auteur « tout ce qui semble nécessaire d'ajouter au milieu didactique extérieur pour le travail des savoirs scientifiques : les connaissances des élèves dont les critères de validité, les problèmes partagés, etc. Le MDIc [milieu didactique intérieur à la classe] étant composé des éléments explicitement partagés par la classe, il comprend essentiellement des textes oraux ou écrits» (p. 49). « Espace de jeu de la problématisation » (qui peut aussi avoir lieu dans le «milieu didactique intérieur à l'élève ») le MDIc permet une « exploration plus large et nécessairement verbalisée du monde des possibles », il a aussi « à voir avec la mémoire didactique » (p. 51 et 52).

- Enfin, en didactique du français, ThevenazChristen, s'inscrivant dans la perspective de l'interactionnisme social, souligne au regard de sa spécificité disciplinaire combien « le milieu didactique inclut la recontextualisation des genres de textes et des unités linguistiques à enseigner " (2002, p. 56) dans un contexte de médiation ce qui l'amène à renoncer de son coté, au vocable « adidactique » (p. 51).

Par ailleurs, au sein même de la théorie des situations didactiques, le modèle du « milieu adidactique antagoniste du sujet » a fait l'objet de remaniements. Les difficultés rencontrées par les chercheurs pour dégager et décrire, lors des études en classe ordinaire, des épisodes relevant d'un « milieu antagoniste » au sens initialement élaboré dans le cadre des ingénieries didactiques, les ont amenés comme nous venons de le 
voir à proposer des extensions au concept initial selon différents qualificatifs (« milieu allié »vs « milieu antagoniste »; « milieu potentiel » vs « milieu effectif »; «assortiments [de milieux] didactiques »). Ces extensions ont entraîné des glissements de sens (Salin, 2002, p. 120 à 123). Pour notre part, nous les considérons comme participant aux « développement d'une ceinture protectrice ${ }^{31} »$ visant à préserver le « noyau dur » du programme de recherche (Lakatos, 1974) initié par Brousseau. Ce dernier a rendu compte des assujettissements subis ou acceptés par l'enseignant lorsqu'il est en situation de réguler un milieu compte tenu des paradoxes du contrat didactique (dévolution et institutionnalisation). Leur formalisation en termes de stratégies et de types de contrats contribue à soutenir l'idée que « le travail didactique $\mathrm{du}$ professeur consiste essentiellement à réguler et changer des contrats didactiques de façon à maintenir des équilibres et des conditions optimales et non pas à appliquer contre vents et marées une méthode aussi sophistiquée soit-elle »(Brousseau, 1996, p. 28).

\section{En conclusion: l'absence d'une théorie unifiée}

Au final, apparaît une grande diversité des qualificatifs utilisés par les chercheurs pour rendre compte des phénomènes qu'ils observent, diversité certes liée aux spécificités disciplinaires, mais qui n'est pas non plus étrangère aux cadres théoriques de référence privilégiés : théorie des situations, théorie anthropologique, apprentissage par problématisation, interactionnisme social. Ces différents arguments invitent à beaucoup de prudence dans l'emprunt et l'utilisation des concepts d'une didactique à une autre. Nous retenons de cette brève revue que si un nombre important de didacticiens ont travaillé la question du milieu, nous ne disposons toutefois pas d'une théorie unifiée ou même partagée. Malgré tout, il ressort de cette discussion que cette notion est incontournable dès lors que l'on veut décrire les pratiques d'enseignement et d'apprentissage. Ce constat nous conduit ici à revenir sur son statut théorique à la lumière du développement des travaux comparatistes en didactique.

\section{Vers une contribution à la théorisation du milieu}

Dans la discussion qui suit nous postulons que la théorie de l'action didactique conjointe (Mercier,
Schubauer-Leoni, \& Sensevy, 2002 ; SchubauerLeoni \& Leutenegger, 2005; Schubauer-Leoni et al., 2007; Sensevy \& Mercier, 2007) est un bon candidat pour élaborer un cadre fédérateur eu égard aux problématiques de milieu et de mésogenèse. Comme ces auteurs, nous pensons nécessaire de décrire l'action didactique en considérant le milieu à la fois dans ses aspects institutionnels (soulignés par Chevallard) et ses aspects situationnels (théorisés par Brousseau). Nous nous sentons confortés dans cette position par l'argumentation développée par Perrin-Glorian (1999) sur la compatibilité de ces deux théories, même si cette dernière est contestée par Joshua (1996a). La proposition de Sensevy (2007, p. 23-25) visant dans sa formalisation de l'action didactique conjointe, à considérer le milieu à la fois « comme contexte cognitif de l'action » et comme « système antagoniste » nous semble rejoindre ce point de vue. Il reste que le caractère « antagoniste » du milieu mérite discussion, d'autant que ce dernier auteur souligne volontiers que le milieu comme système antagoniste modélisant le savoir au sein d'une situation adidactique, ne peut se suffire en lui-même.

Pour notre part, nous considérons qu'une ambiguité subsiste. En effet, si nous considérons fructueux - dans une perspective comparatiste - de retenir l'idée fondatrice introduite par Brousseau ${ }^{32}$, nous nous interrogeons sur la pertinence du vocable « antagoniste ». Sans être toujours antagonistes ${ }^{33}$, les rétroactions du milieu (dans ses dimensions matérielles, conceptuelles, symboliques, sociales, sémiotiques, etc... évolutives) sont cependant susceptibles de créer des épisodes « d'adidacticité ${ }^{34}$ » soutenant l'étude des savoirs par certains élèves (Mercier, 1992, 1997, 1998) comme par exemple pour Mélina ou Ayoub dans certains extraits de notre étude. C'est pourquoi, nous suggérons de découpler du concept de « milieu » les termes « antagoniste » et « adidactique », au profit de celui « d'adidacticité » qui, selon nous, maintient le sens profond porté par la théorisation broussaldienne. $\mathrm{Au}$ fil des transactions didactiques, le processus d'élaboration conjointe du milieu didactique par le professeur et les élèves est susceptible de créer, du fait des rétroactions qui en découlent, les conditions d'un engagement " proprio motu» de l'élève dans la situation (Sensevy, 2007, p. 24). Ce processus - au principe même de la dévolution - est une condition nécessaire à l'émergence d'un épisode d'adidacticité 
dans la biographie didactique d'un élève (Mercier, 1998). Il n'est cependant jamais garanti.

Nous pensons que cette position épistémologique n'est pas étrangère aux réflexions et controverses actuelles sur les thèmes de l'action située dans les investigations en classe (cf. les différentes contributions in Baeriswyl \& Thévenaz, 2001) et plus largement, des liens entre «sujets, activités, environnements » (Barbier \& Durand, 2006). Pour développer cette discussion nous reviendrons tout d'abord sur les liens entre contrat et milieu afin de marquer la solidarité de ces deux notions. Nous discuterons ensuite des problèmes posés par les modélisations en termes de structuration verticale (Margolinas, 1995) ou horizontale (Orange, 2007) en suggérant que ces approches privilégient plutôt des états que des dynamiques. Ce point nous permettra d'avancer sur l'intérêt d'une reconceptualisation des notions de milieu et de mesogenèse pour capturer des configurations de trajectoires possibles dans le fonctionnement des systèmes didactiques. Enfin, nous terminerons en montrant qu'un pas pourrait être franchi en articulant davantage, au sein de la théorie, les liens entre activité et action didactiques.

\section{Milieu et contrat : des notions solidaires}

Il est acquis dans les théorisations didactiques que milieu et contrat sont des notions articulées même si des débats existent entre didacticiens comparatistes sur ce qui, de l'une ou de l'autre, est première. Pour Johsua (1996a) le milieu comprend le contrat; pour Mercier (2002) il est donné par contrat (p. 144). Cette discussion nous semble devoir être dépassée du fait de la codétermination du processus mésogénétique par les dimensions institutionnelles et situationnelles concourant à son actualisation in situ. Nous pensons en effet possible de produire une description de cette dynamique en articulant en quoi elle relève à la fois des usages pratiques sédimentées au sein de l'institution actualisant les éléments pérennes du contrat didactique (Mercier, 1992) et les phénomènes contractuels différentiels à l'œuvre lors des interactions en classe (Schubauer-Leoni, 1996; Sarrasy, 1996). La solidarité des notions de milieu et contrat s'exprime par le fait qu'à tout état de la mésogenèse, correspond un état du partage des responsabilités entre le professeur et les différents élèves (topogenèse) en lien avec l'avancée ou la stagnation du temps didactique (chronoge- nèse) eux-mêmes dépendant des cadres épistémique (relatif au savoir en jeu) et interactionnel (forme des échanges) qui en structure la dynamique. Le travail scientifique consiste alors nous semble-t-il à identifier des configurations (c'est-à-dire des organisations singulières de formes régulières) permettant de rendre compte du fonctionnement du système, comme nous avons tenté de le faire dans en EPS et en physique en contexte difficile. Cette remarque nous amène à revenir sur les modélisations s'attachant à décrire la structuration du milieu.

\section{Les écueils des modélisations en termes de} structuration du milieu

Nous avons déjà pointé que confronté à l'étude des classes ordinaires, le concept de milieu avait fait l'objet de remaniements sous forme de modélisations : verticale, dite en « oignon » (Brousseau 1990, p. 319; Margolinas, 1995, p. 96); ou horizontale (Orange, 2007). Pour Mercier, il s'agit, pour ce qui est de la modélisation de Margolinas, d'une « technique permettant l'analyse conjointe des milieux de l'activité des élèves et du professeur » (2002, p. 114). La modélisation horizontale développée par Orange nous semble répondre à un souci identique relativement à l'apprentissage par problématisation. Les auteurs de ces modélisations considèrent qu'elles facilitent la description des processus. Pour notre part, nous considérons que ces propositions, si elles permettent de saisir des états au sein de dynamiques, sont encore insuffisantes à en rendre compte. Ainsi, le «feuilletage » des milieux pour reprendre l'expression que nous utilisions précédemment nous paraît avoir comme conséquence l'oubli des trajectoires qui se traduisent comme le suggère notre étude en EPS et physique par des avancées, des stagnations $\mathrm{du}$ processus d'enseignement et d'apprentissage, ou encore des évolutions différentielles selon les élèves (comme en physique lorsque, pour certains élèves, des éléments de l'environnement matériel viennent perturber le milieu initialement pensé par le professeur, cf. extrait 2), etc. Mais au-delà, en tant que modèles pour l'analyse didactique in situ, nous questionnons aussi leur économie ${ }^{35}$ en ce qu'ils tendent à démultiplier les descripteurs des milieux activés ou effectifs (Comiti, Grenier, \& Margolinas 1995; Perrin-Glorian, 1999; Perrin-Glorian \& Hersant, 2003; Orange, 2007). 


\section{LE MILIEU DIDACTIQUE : D'UNE ÉTUDE EMPIRIQUE EN CONTEXTE DIFFICILE À UNE RÉFLEXION SUR LE CONCEPT}

Chantal Amade-Escot \& Patrice Venturini

\section{S’intéresser aux dynamiques}

Cette difficulté nous semble pouvoir être levée dès lors que l'on opte pour une approche mettant l'accent sur la co-construction du milieu (Johsua \& Félix, 2002) ou sur les dimensions conjointes de l'action mésogénétique du professeur et des élèves (Schubauer-Leoni \& Leutenegger, 2005; Sensevy $\&$ Mercier, 2007). Moins que la mise en évidence des bifurcations ou des feuilletages du milieu, moins que des analyses de positions, l'avancée théorique consiste à envisager les processus en terme de mésogenèse, selon une approche dynamique permettant d'identifier des « configurations de trajectoires possibles » en articulant différentes échelles de temporalités didactiques : le temps long de la séquence ou du cycle, celui de la séance, enfin le temps court de l'analyse micro-didactique (Badreddine, 2009; Leutenegger, 2008; Schubauer-Leoni et al., 2007; Tiberghien et al., 2007). Dans notre étude, ces configurations s'actualisent dans le fait qu'en badminton les régulations du professeur ne sont intelligibles que si on les rapporte à son intention didactique au-delà de l'épisode ou de la séance : apprendre à déplacer son adversaire pour plus tard attaquer l'espace libre; en physique en raisons des liens entre les séances en demi-groupe et celle en classe entière par rapport à la question de la vision des objets.

Mais s'intéresser aux dynamiques mésogénétiques, c'est aussi prendre en considération les déterminations du jeu didactique (au sens de Sensevy, 2007 , p. 37) c'est-à-dire d'une part, le contexte (ici le collègue situé en "réseau ambition réussite ») et d'autre part, l'épistémologie pratique des professeurs :

- Comme nous l'avons souligné, les contraintes liées au contexte difficile ne sont pas sans effets sur l'action didactique conjointe. Par exemple en physique, il est déterminant que le travail soit totalement conduit en classe car, selon l'enseignante, il est illusoire de proposer aux élèves qu'ils continuent l'étude à la maison (entretien ante) d'où les accélérations observées dans la séance afin de « boucler » les différentes tâches proposées dans la fiche élève. De même, il convient de prévenir tout dérapage, ce qui induit des formes de contrôle non sans effet sur le processus mésogénétique : ne jamais tourner le dos aux élèves et donc ne pas écrire au tableau; tout leur dicter, notamment les points sur lesquels il importe de laisser des traces précises (sous forme d'institutionnalisations dictées systématiques). En EPS, le fait de passer de groupe en groupe, pour s'assurer de la mise en place du travail et pour éviter tout comportement déviant, se concrétise par la reprise systématique d'un groupe à l'autre des points clés du savoir attendu (les 19 occurrences d'injonctions sur la hauteur à donner au volant, cf. supra) ce qui a pour conséquence de renforcer le topos en surplomb adopté par l'enseignant.

- Les sous-bassement épistémologiques de l'action professorale en tant que théories plus ou moins implicites des savoirs enseignés (Sensevy 2007) sont aussi essentiels à la compréhension des phénomènes mésogénétiques. Ainsi, l'attachement du professeur d'EPS à la dimension stratégique du badminton (entretien ante) intervient dans l'agencement du milieu initial (un jeu de duo avec déplacement du joueur opposé); d'une façon analogue, le souci de l'enseignante de physique d'élaborer avec ses élèves des raisonnements à partir de situations expérimentales l'amène à produire des énoncés afin que les élèves travaillent dans le milieu considéré par elle adéquat, comme l'ont montré aussi Sensevy et Quilio (2002). Dans les deux cas, nous avons souligné combien ces deux professeurs tentent de ne rien céder relativement à leur épistémologie professionnelle ambitieuse eu égard aux usages décrits dans la littérature en contexte difficile.

Ces éléments plaident pour une approche du milieu en tant que processus impliquant professeur et élèves. Nous considérons que l'approche comparatiste, mettant au centre de la théorisation les aspects de co-construction (Johsua \& Félix, 2002) ou d'action conjointe (Schubauer-Leoni \& Leutenegger, 2005; Sensevy \& Mercier, 2007) propose une re-conceptualisation des notions de milieu et de mésogenèse permettant d'intégrer l'idée d'adidacticité sans pour cela lier cette dernière à la mise en place d'un système antagoniste modélisant le savoir au sein d'une situation $\mathrm{ad} \mathrm{hoc}{ }^{36}$. Ce point de vue nous amène à revisiter les rapports entre action et situation didactiques.

D’une manière générale, nous considérons que le concept de situation rend compte d'une construction du/des sujet/s à partir des interprétations/significations (pouvant être, ou non, partagées) qu'il/s donne/ nt aux éléments de l'environnement au sein d'une 
institution ${ }^{37}$ les particularisant. L'adoption de cette orientation suppose quelques conventions précisées ci-après. L'environnement fait (ou non) situation pour le sujet et/ou pour un ensemble de sujets d'une institution. Dans ce contexte, action et situation se déterminent réciproquement, la situation contribue à produire l'action, l'action contribue simultanément à la redéfinition de la situation. Plus précisément pour ce qui nous concerne, l'action conjointe du professeur et de/s élèves contribue à produire et à redéfinir le milieu de l'étude. Nous rejoignons ainsi Schubauer-Leoni et al. (2007) lorsqu'elles indiquent que les systèmes de tâches proposées par le professeur peuvent "faire situation », à charge pour le didacticien d'identifier les conditions qui la constituent comme milieu didactique.

Il reste cependant quelques zones d'ombre dans cette approche du processus mésogénétique. Nous les situons justement dans l'intervalle ouvert par les formalisations existantes : celle qui le théorise en termes de co-construction (Johsua, 1996a; Johsua \& Félix, 2002); celle qui l'aborde en termes d'action conjointe (Schubauer-Leoni \& Leuteneger, 2005 ; Schubauer-Leoni et al., 2007; Sensevy, 2007). Nous faisons l'hypothèse que derrière la différence de formulation émerge une question épistémologique encore insuffisamment travaillée par les didacticiens comparatistes : celle des relations entre activité et action didactiques. Sans avoir la prétention de traiter de façon exhaustive cette difficile question (pour une synthèse, voir Bronckart, 2004), nous pensons nécessaire de poursuivre le débat initié par SchubauerLeoni et al. à propos des enracinements de la théorie de l'action didactique conjointe au sein des sciences humaines et sociales (2007, p. 52 à 58) et proposons quelques pistes d'articulation entre théorie de l'action $^{38}$ et analyse de l'activité en didactique.

\section{Considérer que l'activité donne épaisseur à l'enquête actionnelle}

Il s'agit ici d'avancer prudemment quelques arguments relatifs à notre position. Les travaux menés en contexte d'enseignement difficile au sein de notre équipe (Debars, 2005, Debars \& Amade-Escot, 2006, Monnier \& Amade-Escot, 2009, Venturini \& AmadeEscot, soumis) suggèrent que les difficultés du maintien de la relation didactique, dont la conséquence possible est un effacement des savoirs, relèvent de configurations traduisant (sous des formes à chaque fois singulières) certaines régularités dans les dynamiques mésogénétiques observées. A été ainsi mis en évidence une certaine " fragilité » des milieux didactiques en lien avec un topos surplombant endossé par les professeurs et une conduite très serrée de la chronogenèse. Les recherches soulignent en outre, comme dans l'étude comparée d'EPS et de physique de cet article, que les enseignants agissent - en contexte difficile peut-être plus encore qu'ailleurs - dans une interférence d'intentions qui les contraint à faire face à de multiples enjeux en tension et en lien avec l'avancée du savoir. Pour faire face à ces contraintes, ils mobilisent des figures d'action canonique (Bronckart $\&$ Bulea, in Barbier \& Durand, 2006) contextualisées (que l'on peut rapprocher des « i-genres » identifiés par Peltier-Barbier, 2004, cf. note 20) en même temps que des ajustements singuliers en relation avec l'évolution locale et située des interactions didactiques. Ils sont ainsi continuellement conduits à récupérer des situations qui dérivent. Ces constats, théorisés en s'inspirant de Clot (2001) en termes « d'activité didactique empêchée » (pour un développement, Monnier $\&$ Amade-Escot, 2009), invitent à repenser les rapports entre « activité » et « action». En tout cas, il apparaît nécessaire d'aller plus loin que l'assertion qui consiste à les poser comme compatible (Sensevy, 2007, note 4, p. 14) même si nous y souscrivons.

Convaincus à la suite d'autres didacticiens (Schubauer-Leoni et al., 2007) que dans le modèle d'analyse des pratiques d'enseignement et d'apprentissage des savoirs, "la mésogenèse en tant que construction de la référence est première »(p. 58), nous en réexaminons ses liens avec les phénomènes contractuels sous-jacents à la lumière des filiations portées par les notions de «co-construction» ou « d'action conjointe ». L'identité apparente des formulations ( « co-construction » ou « action conjointe ») mérite en effet que l'on s'y arrête au regard des notions d'activité et d'action. Pour ce faire, nous retenons comme y invitent les auteures, les éléments d'articulation proposés par Bronckart (2004, p. 111). Considérant que les conditions d'emploi et le signifié des termes « activité » ou « action » sont multiples et hétérogènes, ce dernier leur attribue un statut interprétatif de lecture de l'agir. L'activité, en référence à Leontiev, y a un caractère premier, organisant et régulant les interactions des individus avec le milieu. Elle 


\section{LE MILIEU DIDACTIQUE : D'UNE ÉTUDE EMPIRIQUE EN CONTEXTE DIFFICILE À UNE RÉFLEXION SUR LE CONCEPT}

Chantal Amade-Escot E Patrice Venturini

implique les dimensions motivationnelles et intentionnelles mobilisées au niveau collectif en termes de finalités socialement validées et s'exprime dans des figures canoniques de l'agir (concrétisées au niveau des usages partagés par les enseignants d'une même discipline, ou par les équipes d'un même type d'établissement par exemple). L'activité inclut ainsi les dimensions institutionnelles aux principes de l'agir. L'action pour sa part, désigne une lecture de l'agir impliquant, selon Bronckart, les mêmes dimensions (intentions et motifs) en ce qu'elles sont mobilisées et portées par la personne singulière dans la situation.

À cette distinction, nous pensons utile d'adjoindre la question du développement (et donc de la temporalité) qui positionne l'activité dans le temps long de l'histoire collective et de la culture (au sens vygotskien) alors que le tempo de l'action la situe dans l'ici et le maintenant de la situation en cours. Cette double distinction (collectif/individuel et temporalité du développement/tempo de la situation immédiate) nous permet de revenir sur les notions de « co-construction du milieu » et « d'action conjointe professeur-élèves » en montrant les nécessaires liens entre activité didactique et action didactique sous-jacents à ces formulations.

Johsua et Félix (2002), quoique n'abordant pas explicitement la question des liens entre ces concepts, mettent l'accent dans leur théorisation du « milieu pour l'étude » sur sa dimension institutionnelle (telle que théorisée par Chevallard, 1992). Se proposant de « traiter à égalité les objets descriptibles en positifs et les ignorances » (p. 91), ils inscrivent nous semblet-il, leur modélisation dans une perspective développementale, incluant la dimension historico-culturelle des rapports au savoir tels que portés par la mémoire de la classe, les usages professionnels, l'épistémologie scolaire en vigueur, sans pour cela laisser de côté les nécessaires ignorances à travailler. Nous postulons que la dimension développementale, soutenue par le concept d'activité (concept cependant non explicitement convoqué) est prépondérante dans la formalisation du « milieu pour l'étude » (Johsua, 1996a; Johsua \& Félix, 2002) d'où la formulation retenue de « co-construction du milieu » impliquant l'idée de co-activité des agents. Cette référence implicite à Vygotski est toutefois développée par Johsua (1996b) à propos du concept de contrat didactique et des phénomènes institutionnels de longue portée temporelle qui y sont associés : « les rapports aux savoirs, dans une tradition scolaire donnée se bâtissent dans un cadre qui dépasse celui du rapport à un savoir donné, objet apparent de la relation hic et nunc» (Ibid, p. 155). La position théorique du «milieu pour l'étude » inclut donc selon nous l'activité dans ses dimensions historique et culturelle comme étant au principe des actions observables dans l'ici et maintenant des séquences observées.

De leur coté, les théoriciens de l'action didactique conjointe (Schubauer-Leoni et al., 2007 ; SchubauerLeoni \& Leutenegger, 2005; Sensevy, 2001, 2007) s'attachent davantage à l'ici et maintenant de l'action conjointe en décrivant les aspects pragmatiques de la mésogenèse, notamment par le biais des énoncés langagiers (Sensevy \& Quilio, 2002), même si la notion de milieu institutionnel est fortement présente dans leur propos (cf. le milieu comme contexte cognitif de l'action chez Sensevy, 2007, p. 23; ou encore le statut de l'institution chez Schubauer-Leoni et al., 2007, p. 57). Il s'agit pour ces auteurs - sans perdre de vue les conditions historiques et collectives de production des œuvres à l'étude dans l'institution scolaire - de situer plus précisément les conditions dans lesquelles sont produit les savoirs, en tant que processus micro-historiques liés aux interactions didactiques (Forget \& Schubauer-Leoni, 2008). Cette posture amène ces dernières à suggérer de porter le regard sur « la transposition mésogénétique interne » à savoir « l'étude de la transformation qui peut s'opérer entre l'interprétation que l'enseignant fait des différents milieux constitués et l'interprétation que les élèves font de ces mêmes milieux, sous couvert du contrat didactique » (2008, p. 186). Nous notons là une attention plus grande aux aspects situationnels et interactionnels, qui s'attache à identifier « la manière dont les objets de savoir mis à l'étude et enchâssés in situ dans des dispositifs ou des tâches d'apprentissage sont re-configurés et co-construits par les partenaires de la relation didactique à travers leurs interactions »(Amade-Escot, 2007, p. 16).

Ayant pointé les différentes sensibilités et focales qui sous-tendent les formulations retenues par les didacticiens comparatistes pour décrire le processus de genèse d'un milieu didactique, soulignons toutefois que tous s'accordent à considérer le processus mesogénétique selon diverses échelles temporelles. Cette assertion nous invite à ne pas en réduire la dynamique d'élaboration à l'ici et maintenant de 
l'action immédiate. Nous considérons de ce fait qu'il n'y a pas d'opposition de fond entre « milieu pour l'étude » et «mésogenèse »; entre " co-construction du milieu » et "action mésogénétique conjointe » en ce que ces deux approches mettent au centre de leur modélisation le milieu en tant que « construit permanent » (Chevallard, 1992) au regard de phénomènes situés au-delà et en deçà des observations. Les différences de formulation témoignent selon nous des types de centrations opérées par les auteurs, dont nous souhaitons relever le caractère complémentaire. Pour autant, ces différences pointent la nécessité théorique de continuer à clarifier les liens entre activité et action didactiques.

Pour notre part, nous les envisageons à partir de l'étude « du » didactique en contexte difficile en ce qu'il impose de mettre en enquête l'activité didactique (et ses empêchements) à partir de l'action conjointe incertaine du professeur et des élèves, cette dernière se trouvant en retour éclairée par la mise au jour de "l'activité didactique empêchée " (Monnier $\&$ Amade-Escot, 2009). L'étude comparative de l'enseignement de l'EPS et de la physique présentée dans cet article ainsi que la discussion esquissée autour du concept de milieu didactique qui en a suivi conduisent à considérer qu'au sein du système de descripteurs de l'action didactique conjointe que constitue le triplet de genèses (méso-, topo-, chrono-) celui de mésogenèse parce qu'il est en mesure de rendre compte du tissage et de l'articulation de l'activité didactique (dans ses dimensions historique, épistémologique, culturelle et institutionnelle) et de l'action didactique (dans ses dimensions situationnelle et interactionnelle) est décisif. Dans ce cadre, il apparaît heuristique de penser les relations entre milieu et mésogenèse comme outil de caractérisation du fonctionnement des systèmes didactiques permettant de saisir à l'échelle des micro-histoires les «manières de voir comme " des élèves ou du professeur sans perdre de vue leur inscription dans le temps plus long du développement et de l'apprentissage. C'est dans cette dynamique de transformations conjointes qu'émergent les nouveaux rapports personnels aux savoirs mis à l'étude, et leur adéquation (ou non) avec les rapports aux savoirs institutionnels visés. Une telle perspective place résolument la théorie didactique parmi les approches situées de l'enseignement et de l'apprentissage (Amade-Escot, 2007, Amade-Escot \& O'Sullivan, 2007; Sensevy, 2008) sans pour cela sacrifier à un situationnisme radical mais au contraire en prenant résolument parti - au sein des controverses qui les traversent relativement à la part dévolue à l'individuel ou aux rôles respectifs de la culture et d'autrui dans les processus de développement (cf. Moro, in Baeriswyl \& Thévenaz, 2001) - pour les seconds.

\section{En guise de conclusion ouverte ...}

Nous avons initié cette réflexion à partir d'une recherche visant à décrire et expliquer les pratiques didactiques des enseignants en contexte difficile en postulant qu'elle nous permettrait de saisir certains aspects génériques des processus à l'œuvre dans l'action didactique conjointe professeur élève. L'analyse comparée de séquences d'EPS et de physique a mis en évidence que ce qui était le plus problématique, en ce que susceptible de rendre compte d'un affaiblissement de l'épaisseur épistémologique des savoirs mis à l'étude dans ces contextes (dont rend aussi compte un ensemble de recherches en Sciences de l'Éducation évoqué en introduction) relève de la manière dont professeur et élèves, conjointement, contribuent à faire émerger des environnements (des milieux) plus ou moins propices à la construction d'une référence commune compatible avec des pratiques de savoirs à l'œuvre dans la culture humaine. Nous avons aussi montré que ces constats sévères devaient néanmoins être nuancés à la lumière des trajectoires, à chaque fois singulières, des systèmes étudiés, en ce qu'ils rendaient compte de phénomènes sans doute génériques, que l'effet de «loupe » du contexte étudié permettait de révéler. C'est ainsi que nous sommes passés de l'étude des évolutions des milieux didactiques en classe d'EPS et de physique dans un collège « ambition réussite " à une réflexion sur le statut théorique du concept de « milieu » dans l'étude en classes ordinaires.

La discussion a permis d'avancer l'idée que le « milieu didactique » est un concept tissant les possibles et les nécessaires aller retour entre l'ici et maintenant de l'action conjointe et les substrats historiques, collectifs et institutionnels qui en déterminent la dynamique. Contre une posture épistémologique trop radicalement « situationniste », la discussion accrédite ainsi l'idée paradoxale - mais magistralement développée dans l'œuvre de Bourdieu - selon laquelle la pratique concilie à la fois la détermination de la conduite et l'intentionnalité de l'action (1997). 


\section{NOTES}

1. Le terme de «milieu difficile» est souvent retenu pour qualifier le contexte des établissements situés notamment en zone d'éducation prioritaire. Toutefois, pour faciliter la lecture et éviter les confusions possibles avec le terme de «milieu didactique», concept central de cet article, nous avons choisi d'utiliser le terme de «contexte difficile» pour désigner cette réalité.

2. Nous nous limitons dans le cadre de cet article aux auteurs utilisant explicitement le concept de «milieu didactique» tel qu'initialement théorisé par Brousseau. Certains chercheurs examinent les questions que nous tentons d'aborder en référence aux théories vygotskiennes (par exemple, Brossard, 2001). Dans cette lignée théorique citons en particulier les travaux de didacticiens comparatistes analysant les contextes d'apprentissage scolaires sous couvert du concept de «communauté discursive» (Bernié, 2002 ; Bernié, Jaubert, \& Rebière, 2005 ; Schneeberger, 2008) pour ne citer que quelques articles. Voir aussi le colloque «Construction des connaissances et langage dans les disciplines d'enseignement» (Jaubert, Rebière, \& Bernié, 2003).

3. Johsua et Félix (2002) ont utilisé cette formulation en l'associant à un point de vue sur le milieu, qui sera repris dans la discussion de la dernière partie. Sauf référence explicite à ces auteurs, la formulation «milieu pour l'étude» s'entend dans cette partie de l'article sans cet arrière plan théorique. Il s'agit simplement pour nous de désigner le milieu par sa fonction.

4. A la rentrée 2006, l'éducation prioritaire en France a été restructurée : d’une logique de «zone» (d'éducation prioritaire) dotée systématiquement de moyens supplémentaires, on passe à une logique de «projet», destinée à «assurer la réussite scolaire de tous les élèves». Cette logique est matérialisée notamment par la création de réseaux «ambition réussite», regroupant avec le collège, les écoles élémentaires et maternelles d'où proviennent ses élèves, ainsi que leurs partenaires. Circulaire 06-058 du 30/3/06 «Principes et modalités de la politique de l'éducation prioritaire».

5. Nous reprenons ici, d'un point de vue micro-didactique, des données recueillies selon la méthodologie décrite dans cet article, dans une classe de $6^{\text {ème }}$ lors d'un master de recherche visant à comparer la pratique d'intervention d'un enseignant d'EPS avec deux classes contrastées ( $6^{\text {ème }}$ et «primo arrivants») de ce collège (Debars, 2005).

6. Dans les documents d'accompagnement pour les classes de $6^{\text {ème }}$, l'expression «gestion d'une cible à libérer puis à exploiter» rend compte de la nécessité pour un joueur de déplacer son adversaire de façon à créer un espace libre où envoyer ensuite le volant dans le but de marquer le point. Les préconisations institutionnelles valorisent cette entrée didactique pour amener les élèves à créer, en position favorable, la rupture de l'échange (thème du programme)

7. L'étude de deux de ces séances a fait l'objet d'une publication. Nous nous y appuyons à certains moments de l'interprétation (Venturini \& Amade-Escot, soumis)

8. Une «frappe de dégagement» dans le vocabulaire du badminton est un geste technique visant à produire une trajectoire du volant suffisamment haute pour permettre au joueur de se donner du temps. Le savoir y afférant met en jeu de façon indissociable des dimensions technique, tactique et stratégique. Ces deux derniers termes renvoient aux aspects décisionnels de l'action (en cours d'action pour ce qui relève de la tactique, anticipées pour ce qui relève de la stratégie).

9. Compte tenu de l'effectif de la classe (15 élèves), cette organisation ( 2 joueurs par terrain) permet que tous les élèves soient en action, à l'exception d'un groupe de 3 garçons «remuants» qui occupent un même terrain. L'enseignant les surveille de façon rapprochée pour éviter tout dérapage : «Farid ... on a dit que quand il y avait des groupes de trois chacun faisait trois minutes»; "vous avez un chrono là-bas trois minutes tu remplaces Youness après trois minutes Youness remplace Ayoub»; "c'est pas difficile quand on veut de s'organiser .. par contre tu t'écartes pour ne pas les gêner».

10. Notamment ceux observés en demi-classe lors des deux séances précédentes.

11. Au double sens «d'entrer dans le vif du sujet» relativement à l'enjeu de savoir et de «vitalité» du milieu didactique portant potentiellement cet enjeu. 
12. Il s'agit pour le joueur (A) qui doit déplacer son partenaire (B) sur le terrain opposé de produire des trajectoires hautes à droite et à gauche, et pour le joueur (B) de produire des trajectoires hautes (pour se donner le temps du replacement) en direction du joueur (A).

13. Dans cette tâche, la «frappe de dégagement», n'est pas seulement un coup d'attaque ou un coup de défense. Elle est la préparation d'un coup d'attaque pour le joueur qui veut prendre l'avantage dans le rapport de force. Mais elle est aussi, dialectiquement un coup défensif pour le joueur qui, sous la pression de l'adversaire, veut ralentir le jeu pour rééquilibrer le rapport de force. L'intérêt même de cette tâche de duo est de positionner le double enjeu possible de la «frappe de dégagement» en gardant une des caractéristiques «grammaticales» du jeu de badminton, c'est-à-dire l'aspect dialectique du jeu et les différentes formes de l'alternative décisionnelle

14. Nous reviendrons sur cette question en dernière partie de l'article.

15. Nous attirons l'attention du lecteur sur le fait que contrairement à ce qu'une lecture rapide du verbatim de l'extrait 1 pourrait laisser penser, les élèves ont bien saisi le jeu auquel le professeur les invite comme en témoignent leurs réponses (cf. tdp 14, 16, 17). Par ailleurs, rappelons (cf. section traitement des données) que la distinction entre source primaire et source secondaire a été mise à l'étude lors de la séance précédente. Soulignons enfin, que la compréhension de cette distinction est attestée dans la recherche par des entretiens menés avec les élèves (non rapportés dans cet article).

16. Dans cet article, nous retenons le point de vue développé par Schubauer-Leoni et al. (2007), relativement à l'observation du didactique ordinaire. La tâche renvoie aux conditions du travail proposé par le professeur à ses élèves, au sens premier des «choses à faire» (pour une discussion dans la théorisation de l'action conjointe, voir p. 60-62).

17. Les éléments de régulation de l'enseignant dans cet épisode, tout comme l'analyse de son discours lors des entretiens ante et post séances, nous permettent de lever l'idée d'un «manque à savoir didactique». Cette incise vise à ne pas rabattre les observations effectuées sur un jugement de faible compétence, nous pensons au contraire que c'est dans l'action didactique conjointe en contexte difficile que doivent être éprouvées les raisons de ces comportements

18. Voir en particulier note 2 .

19. Nous renvoyons le lecteur à l'analyse comparative de l'action didactique conjointe de cette même enseignante en demi-classe et classe entière (Venturini \& Amade-Escot, soumis)

20. Pour décrire l'enseignement des mathématiques en réseaux d'éducation prioritaire, les auteurs - dans le cadre d'une approche ergonomique des pratiques didactiques - sont amenés à préciser deux composantes concourant au genre du métier enseignant : «les i-genres (qui rendent compte de la mission d'instruction) et les e-genres qui rendent compte de la mission d'éducation)». Les i-genre constituent des systèmes de réponses cohérents et stables en relation avec les apprentissages scolaires. Ils sont caractérisés par un ensemble d'indicateurs : «recours plus ou moins fréquent à des problèmes, places respectives accordées au collectif et à l'individuel, place laissée à l'initiative des élèves, modes d'étayage mis en œuvre régulièrement» (Butlen \& Peltier-Barbier, in Peltier-Barbier, 2004, p. 65 à 68).

21. Contrairement, pour ce qui est de la physique, à ce que nous avons pu observer en demi-classe.

22. S'il est possible de considérer que les dispositifs produits par les deux professeurs ne puissent «faire milieu» en raison d'une faiblesse de leur agencement à la fois en termes de fonctionnement et de significations, nous résistons cependant à établir de façon assurée cette interprétation. Nous rejoignons Schubauer-Leoni et al. (2007, p.87) qui indiquent en substance qu'à l'échelle de la micro-histoire de la situation, ou même de la séance, les observables construits par les didacticiens ne peuvent que mettre en évidence des manques à savoir didactique des professeurs. A une échelle de temporalité plus large (dans cette recherche, incluant l'ensemble du corpus c'est-à-dire 3 séances d'EPS et de physique portant sur le même thème) nous pouvons attester des acquisitions des élèves. C'est d'ailleurs cette question que nous développons en théorie dans la suite de cet article lorsque nous esquissons une discussion sur les nécessaires articulations à construire entre activité et action didactiques. 


\section{LE MILIEU DIDACTIQUE : D'UNE ÉTUDE EMPIRIQUE EN CONTEXTE DIFFICILE À UNE RÉFLEXION SUR LE CONCEPT}

Chantal Amade-Escot E Patrice Venturini

23. Ajoutons à cette interprétation que les phénomènes dont nous rendons compte ne sont pas exclusivement liés aux conditions de l'enseignement en "classes difficiles», mais relèvent de phénomènes assez génériques, observables sous des formes sensiblement identiques dans toutes les classes. Nous pointons simplement qu'ils sont davantage exacerbés en contexte difficile lorsque, face à une situation critique d'un point de vue didactique (liée à certaines difficultés relatives au savoir visé), le professeur est amené à arbitrer entre des tensions contradictoires. Cet arbitrage, dans les extraits que nous présentons, prend la forme d'un contrôle topogénétique du savoir au détriment de la dévolution (constat assez récurrent si l'on en croit la revue de question esquissée en début d'article).

24. Ce terme réfère au fait qu'en contexte difficile plus qu'ailleurs, les élèves, au fil des interactions, sont susceptibles de déserter l'espace didactique ce qui compromet la co-construction du milieu.

25. Citons sans prétendre à l'exhaustivité en français (Thevenaz-Christen, 2002), en EPS (Amade-Escot, 2005 ; Amade-Escot, Verscheure, \& Devos, 2002 ; Roustan \& Amade-Escot, 2004 ; Rilhac, 2007 ; Thépaut \& Léziart, 2007)n en sciences (Joshua, 1996a ; Orange, 2007 ; Tsoumpélis, 2005).

26. Modélisation qui initialement s'inscrit dans un paradigme de recherche d'ingénierie didactique présupposant l'existence d'une situation fondamentale et la recherche de milieux adidactiques associés à un savoir (Perrin-Glorian, 1994, Salin, 2002) et traitant d'un certain type d'empirie dont rendent compte les recherches menées au COREM (Centre pour l'Observation et la Recherche sur l'Enseignement des Mathématiques) de Talence.

27. Identifié en EPS sous le terme «magie de la tâche» (pour une discussion, Amade-Escot, 2005)

28. Les extraits 2 et 6 de la séance de physique en attestent.

29. Tsoumpélis (2005) précise auparavant (p. 206), en reprenant des analyses de Chevallard, que l'expérience en mathématiques n'a pas le même statut qu'en physique : «il ne s'agit pas d'une expérience sur le système à modéliser, mais d'une expérience sur le modèle». Aussi, lorsque l'élève «entre en interaction avec un milieu mathématique [...] les aspects pertinents d'un milieu matériel ne sont que ses aspects mathématiques» (p. 216), ces dernier étant seuls "pertinents pour la situation adidactique» (p. 217). Son analyse, en accord avec les aspects épistémologiques précédents l'amène à considérer le milieu en mathématiques comme «interne aux mathématiques». En physique, le milieu comporte, selon ce auteur, deux composantes, une composante mathématique, qui est interne à la physique, "indépendante des explications possibles des processus physiques» et une composante matérielle, correspondant au monde réel, donc externe de ce fait à la physique, ayant son propre mode de fonctionnement, et servant de référence «aux prédictions de faits qualitativement nouveaux» (p. 217).

30. Indiquons, à cette occasion que lorsque «Brousseau évoque ses sources, relativement au concept de milieu, c'est au logicien Lorenzen qu'il se réfère et non au 'milieu' des biologistes» (rapporté par Salin, 2002, p.123). Les différentes sources convoquées par les didacticiens suivant les disciplines ne sont pas sans poser de redoutables questions épistémologiques à la théorisation « du » didactique.

31. Nous soutenons l'idée que ces remaniements illustrent ce que Lakatos (1974) développe relativement à l'évolution des programmes de recherches. Le noyau dur théorique du programme (ici le concept de «milieu adidactique antagoniste de l'élève») fédère les chercheurs s'en réclamant. Selon cet épistémologue, la remise en cause du noyau dur est toujours problématique. En conséquence, le développement du programme porte sur la ceinture protectrice et procède par ajout d'éléments heuristiques, de concepts, etc. Ce sont les avancées de la ceinture protectrice qui sont soumises au débat et à la réfutation. Les ajouts successifs proposés par les chercheurs en terme de structuration du milieu, d'assortiments didactiques, de milieu allié, etc. (cf. Salin, 2002), apparus lors des travaux menés en classe ordinaire, peuvent être lus, selon nous, comme permettant de ne pas remettre en cause le noyau dur de la théorie.

32. Parce que nous la considérons essentielle.

33. On a vu que certains suggèrent que le milieu didactique soit «allié» (Frégona, citée par Salin, 2002, cette dernière récusant cependant ce terme) pour indiquer que l'élève peut agir dans un tel milieu en mettant en œuvre des solutions algorithmiques, pensées comme évidentes par le professeur. 
34. Ces épisodes peuvent survenir au cours de l'étude, en classe ou même en dehors de la classe, comme l'ont montré Johsua et Félix (2002) à propos du travail personnel à la maison, tout en soulignant les dépendances entre les deux types d'activité.

35. Walliser (1997) considère que l'économie d'un modèle est une caractéristique essentielle à son efficience.

36. Nous ne sous-estimons pas cependant l'intérêt des modélisations de situations adidactiques produites par les recherches d'ingénierie en tant qu'elles ont permis de renouveler radicalement la façon de penser le didactique, tout en précisant les conditions auxquelles doit souscrire une analyse épistémologique du savoir.

37. Institution qui façonne les manières de voir et de penser de ses sujets, au sens de l'anthropologue Douglas (1987)

38. Indiquons d'emblée à propos du terme d'action dans la modélisation proposée que nous récusons avec Schwartz (2001) l'épistémologie solipsiste de l'action rationnelle. Nous nous inscrivons plutôt dans le tournant actionnel critique qui caractérise la réflexion actuelle des sciences de l'éducation relativement aux modèles d'action rationnelle hérités du XIX ${ }^{e}$ et dont rend compte, dans sa diversité, l'ouvrage collectif dans lequel est publié l'article de Schwartz.

Nous remercions les experts pour leurs commentaires qui ont permis de prolonger notre réflexion théorique

\section{RÉFÉRENCES}

Amade-Escot, C. (2002). Etude du travail de l'enseignant d'éducation physique dans la classe : contribution des recherches didactiques à l'analyse des pratiques effectives. In J.F. Marcel (Ed.), Les Sciences de l'Éducation : des recherches, une discipline, (pp.53-78). Paris : L'Harmattan, Savoir et Formation.

Amade-Escot, C. (2005). Milieu, dévolution et contrat didactique : regard de l'éducation physique. In M.H. Salin, P. Clanché, \& B. Sarrazy (Eds.), Sur la théorie des situations didactiques. Questions, réponses, ouvertures. Hommage à Guy Brousseau (pp. 91-98). Grenoble : La pensée sauvage.

Amade-Escot, C. (2007). Le didactique. Paris : Editions Revue EP.S.

Amade-Escot, C., \& O'Sullivan, M. (Eds.) (2007). Research on content in physical education : theoretical perspectives and current debates. Physical Education and Sport Pedagogy [Special Issue], 12(3), 185-203.

Amade-Escot, C., Verscheure, I., \& Devos-Prieur, O. (2002). Agencement des milieux et régulations didactiques : outils d'analyse de l'activité du professeur en éducation physique. Les Dossiers des Sciences de l'Éducation, 8, 87-97.

Amigues, R., Félix, C. \& Saujat, F. (2008). Les connaissances sur les situations d'enseignement-apprentissage à l'épreuve des prescriptions. Les dossiers des Sciences de l'Éducation, 19, 27-39.

Andersson, B., \& Karrqvist, C. (1983). How swedish pupils aged 12-15 years, understand light and its properties. European Journal of Science Education, 5(4), 387-402.

Badreddine, Z. (2009). Étude des décisions chronogénétiques des enseignants dans l'enseignement de la physique au collège; une étude de cas au Liban. Thèse de Sciences de l'Éducation, non publiée, Lyon, Université Louis Lumière. 


\section{LE MILIEU DIDACTIQUE : D'UNE ÉTUDE EMPIRIQUE EN CONTEXTE DIFFICILE À UNE RÉFLEXION SUR LE CONCEPT}

Chantal Amade-Escot E Patrice Venturini

Baeriswyl, F., \& Thévenaz, T. (2001) (Coord.). Eclairages sur la « cognition située » et modélisation des contextes d'apprentissage. Revue Suisse des Sciences de l'Éducation, 23(3), 395-528.

Bakhtin, M. M. (1952/1986). The Problem of Speech Genre. In C. Emerson, \& M. Holquist (Eds.), Speech Genres and Other Late Essays (pp. 60-102). Austin : Universtiy of Texas Press.

Barbier, J.-M., \& Durand, M. (2006). Sujets, activités, environnements. Approches transverses. Paris : PUF, Education et formation.

Bautier, E. (2002). L'enseignement en ZEP et les recherches en didactique du français. Revue Française de Pédagogie, 140, 53-64.

Bautier, E., \& Goigoux, R. (2004), Difficultés d'apprentissage, processus de secondarisation et pratiques enseignantes : une hypothèse relationnelle. Revue Française de Pédagogie, 148, 89-100.

Bernié, J.-P. (2002). L'approche des pratiques langagières scolaires à travers la notion de « communauté discursive » : un apport à la didactique comparée. Revue Française de Pédagogie, 141, 77-88.

Bernié, J.-P., Jaubert, M., \& Rebière, M. (2005). Bases et perspectives d'une évaluation formative des pratiques langagières au service de la construction des connaissances dans les disciplines scolaires. Repères, 31 , 189-210.

Bronckart, J.-P. (2004). Pourquoi et comment analyser l'agir verbal et non verbal en situation de travail. In J.-P. Bronckart (Ed.), Agir et discours en situation de travail (pp. 11-144). Genève : Cahier de la section des sciences de l'éducation, $\mathrm{N}^{\circ} 103$.

Brossard, M. (2001). Situations et formes d'apprentissage. Revue Suisse des Sciences de l'Éducation, 23(3), 423-437.

Brousseau, G. (1986). Fondements et méthodes en didactique des mathématiques. Recherches en didactiques des mathématiques, 7(2), 33-115.

Brousseau, G. (1990). Le contrat didactique : le milieu. Recherches en Didactique des Mathématiques, 9(3), 309-336.

Brousseau, G. (1996). L'enseignant dans la théorie des situations didactiques. In R. Noirfalise \& M.-J. PerrinGlorian (Eds), Actes de la VIIIème école d'été de didactique des mathématiques (pp.3-46). Clermont Ferrand : IREM.

Bourdieu, P. (1997). Méditations pascaliennes. Paris : Seuil.

Butlen, D., Peltier-Barbier, M.-L., \& Pezard, M. (2002). Nommés en REP ? Comment font-ils? Pratiques de professeurs d'école enseignant les mathématiques en REP : contradiction et cohérence. Revue Française de Pédagogie, 140, 41-52.

Carlsen, W.S. (1991). Questioning in classrooms. A sociolinguistic perspective. Review of Educational Research, 61, 157-178.

Chevallard, Y. (1992). Concepts fondamentaux de la didactique: perspectives apportées par une approche anthropologique. Recherches en Didactique des Mathématiques, 12(1), 73-112. 
Chevallard, Y. (2007). Passé et présent de la théorie anthropologique du didactique. Conférence plénière au premier congrès international sur la théorie anthropologique du didactique. In L. Ruiz-Higueras, A. Estepa, \& F. Javier García (Eds.), Sociedad, Escuela y Mathemáticas. Aportaciones de la Teoría Antropológica de la Didáctico (pp. 705-746). Universidad de Jaén.

Clot, Y. (2001). Clinique de l'activité et pouvoir d'agir : Editorial. Education Permanente, 146, 7-16.

Comiti, C., Grenier, D., \& Margolinas, C. (1995), Niveaux de connaissances en jeu lors d'interactions en situation de classes et modélisation de phénomènes didactiques. In Arsac et al. (Eds.), Différents types de savoirs et leur articulation (pp. 92-113). Grenoble: La Pensée sauvage.

Debars, C. (2005). Contribution à l'étude de l'action didactique d'un professeur d'EPS en ZEP, Observations de deux cycles de badminton. Mémoire de Mastère 2 Recherche en Sciences de l'Éducation, non publié, Université de Toulouse Le Mirail, Toulouse.

Debars C., \& Amade-Escot C. (2006). Enseigner le badminton à une classe de primo arrivants : l'incidence du contexte ZEP (zone d'éducation prioritaire) sur la pratique d'intervention. Revue eJRIEPS, 9, 35-43, disponible sur Internet à l'adresse : http://www.fcomte.iufm.fr/CD_RENTREE_2007/rentree/RECHERCHE/ ejrieps/index.php (consulté le 2 mars 2009)

Douglas, M. (1999). Comment pensent les institutions. Paris : La découverte, Collection recherche Mauss.

Engle, R. A., \& Conant, F. R. (2002). Guiding principles for fostering productive disciplinary engagement : explaining an emergent argument in a community of learners' classroom. Cognition and Instruction, 20(4), 399-483.

Forget, A., \& Schubauer-Leoni, M.-L. (2008). Inventer un code de désignation d'objets au début de la forme scolaire. Des productions personnelles à la convention collective. In L. Filliettaz, \& M-L. Schubauer-Leoni (Eds.), Processus interactionnels et situations éducatives (pp. 183-204). Bruxelles: De Boeck, Raisons Educatives.

Gomet, D. (2003). Badminton: de l'élève débutant au joueur confirmé. Paris : Vigot.

Guesne, E. (1983). Children's ideas about light / Les conceptions des enfants sur la lumière. In New trends in Physics Teaching (pp. 179-192). UNESCO vol IV.

Herrenkoh, L. R., \& Guerra, M. R. (1998). Participant structures, scientific discourse, and student engagement in fourth grade. Cognition and Instruction, 16, 431-473.

Jaubert, M., Rebière, M., \& Bernié, J.-P. (2003). Construction des connaissances et langage dans les disciplines d'enseignement. Actes du colloque pluridisciplinaire international [Cd-ROM], Bordeaux, 3-5 avril, France.

Johsua, S. (1996a). Sur la notion de milieu. Document interne. Aix-En-Provence : Centre Interdisciplinaire de Recherches Apprentissage, Didactique, Évaluation [non publié].

Johsua, S. (1996b). Le concept de contrat didactique et l'approche vygotskienne. In C. Raisky, \& M. Caillot (Eds.), Au-delà des didactiques, le didactique : débats autour de concepts fédérateurs (pp.145-158). Bruxelles : De Boeck, Perspectives en éducation. 


\section{LE MILIEU DIDACTIQUE : D'UNE ÉTUDE EMPIRIQUE EN CONTEXTE DIFFICILE À UNE RÉFLEXION SUR LE CONCEPT}

Chantal Amade-Escot E Patrice Venturini

Johsua, S. (2002). Spécificités disciplinaires, spécificités didactiques, vers une didactique comparée. In P. Venturini, C. Amade-Escot, \& A. Terrisse (Eds.), Etude des pratiques effectives : l'approche des Didactiques (pp. 17-24). Grenoble : La pensée sauvage.

Johsua, S., \& Félix, C. (2002). Le travail des élèves à la maison : une analyse didactique en terme de milieu pour l'étude. Revue Française de Pédagogie, 141, 89-97.

Lakatos, I. (1974). Falsification and the methodology of scientific research programmes. In I. Lakatos, \& A. Musgrave (Eds.), Criticism and the growth of knowledge (pp.91-196). Cambridge : Cambridge University Press.

La Rosa, C., Mayer, M., Patrizi, P., \& Vincenti-Missoni, M. (1984). Commonsense knowledge in optics: preliminary results of an investigation into the properties of light. European Journal of Science Education, 6(4), 387-397.

Leutenegger, F. (2003). Etude des interactions didactiques en classe de mathématiques : un prototype méthodologique. Bulletin de psychologie, 56(4), 559-571.

Leutenegger, F. (2008). L'entrée dans une code écrit à l'école enfantine et l'articulation entre le collectif et l'individuel : comparaison de deux études de cas. Education \& Didactique, 2(2), 7-42

Lobato, J., Clarke, D., \& Burns Ellis, A. (2005). Initiating and eliciting in teaching : a reformulation of telling. Journal for Research in Mathematic Education, 36, 101-136.

Mauss, M. (1935). Sociologie et anthropologie. Paris : PUF

Margolinas, C. (1995) La structuration du milieu et ses apports dans l'analyse a posteriori des situations. In C. Margolinas (Ed.), Les débats de didactique des mathématiques (pp.89-102). Grenoble : la pensée sauvage.

Margolinas, C. (1999). Le milieu et le contrat, concepts pour la construction et l'analyse des situations d'enseignement. In R. Noirfalise (Ed.), Analyse de pratiques enseignantes et didactique des mathématiques, Actes de l'université d'été de La Rochelle (pp. 3-16). Clermont-Ferrand : IREM.

Marsenach, J. (1995). EPS au collège et volley-ball. Paris : INRP.

MEN, (1998). Enseigner au collège : Education physique et sportive. Programmes et accompagnements. Paris : CNDP.

MEN, (2005). Programmes de sciences physiques, classe de cinquième, Bulletin officiel de l'Éducation nationale, hors série $\mathrm{n}^{\circ} 05 \mathrm{du} 25$ août 2005.

Mercier, A. (1992). L'élève et les contraintes temporelles de l'enseignement, un cas en calcul algébrique. Thèse de doctorat, non publiée, Université de Bordeaux I, Bordeaux, France.

Mercier, A. (1997). Le milieu et la dimension adidactique des relations didactiques. In J. Brun, F. Conne, \& R. Floris, (Eds.), Interactions didactiques, Analyse de protocoles entre didactique des mathématiques et psychologie cognitive (pp. 5-23). Genève: Actes des premières journées didactiques de la Fouly, Didactique des mathématiques. 
Mercier, A. (1998). Participation des élèves à l'enseignement. Recherches en Didactique des Mathématiques, 18(3), 279-310.

Mercier, A., Schubauer-Leoni, M., \& Sensevy, G. (2002). Vers une didactique comparée. Revue Française de Pédagogie, 141, 5-16.

Monnier, N., \& Amade-Escot, C. (2009). L'activité didactique empêchée : un outil d'intelligibilité de la pratique enseignante en milieu difficile. Revue Française de Pédagogie, in press.

Mortimer, E., \& Scott, P. (2003). Meaning making in secondary science classrooms. Maidenhaed : Open University Press.

Orange, C. (2007). Quel milieu pour l'apprentissage par problématisation en sciences de la vie et de la terre. Education \& Didactique, 1(2), 37-56

Peltier-Barbier M.-L. (2004). Dur d'enseigner en ZEP. Dur pour les élèves, Dur pour les enseignants. Grenoble : La Pensée sauvage, Recherches en didactique des mathématiques.

Perrin-Glorian, M.-J. (1994). Théorie des situations didactiques : naissance, développement, perspective. In M. Artigue, R. Gras, C. Laborde, \& P. Tavignot (Eds.), Vingt ans de didactique des mathématiques en France (pp.97-147). Grenoble : La pensée sauvage, Recherches en didactique des mathématiques.

Perrin-Glorian, M.-J. (1999). Problèmes d'articulation de cadres théoriques : l'exemple du concept de milieu. Recherches en Didactique des Mathématiques, 19(3), 279-322.

Perrin-Glorian, M.-J. \& Hersant M. (2003). Milieu et contrat didactique, outils pour l'analyse de séquences ordinaires. Recherches en Didactique des Mathématiques, 23(2), 217-276.

Rilhac, P. (2007). Actions de l'élève et milieux didactiques : la notion de surassujettissement. Carrefours de l'éducation, 24, 159-182

Rochex J.-Y. (1997). Les ZEP : un bilan décevant. In J.-P. Terrail (Ed.), La scolarisation de la France : Critique de l'état des lieux (pp. 123-140). Paris: La dispute.

Rochex, J.-Y., \& Kheroubi, M. (2004). La recherche en éducation et les ZEP en France. 2 Apprentissage et exercice professionnel en ZEP : résultats, analyses, interprétations (note de synthèse). Revue Française de Pédagogie, 146, 115-190.

Roustan C. (2003). La mise en place d'un milieu pour l'étude d'une activité physique et sportive au CP : le cas du badminton. Thèse de doctorat de Sciences de l'Éducation, non publiée, Université d' Aix-Marseille I, Aix en Provence, France.

Roustan, C., \& Amade-Escot, C. (2004). Une analyse didactique en terme de "milieu pour l'étude" : approche spécifique à l'EPS et dialectique de la co-construction. Revue Suisse des Sciences de l'Éducation, 25(3), 481-204.

Salin, M.H. (2002). Repères sur l'évolution du concept de milieu en théorie des situations. In J.-L. Dorier, M. Artaud, M. Artigue, R. Berthelot, \& R. Floris (Eds.). Actes de la 11 ime Ecole d'Eté de Didactique des Mathématiques (pp. 111-124). Grenoble: La pensée Sauvage. 


\section{LE MILIEU DIDACTIQUE : D'UNE ÉTUDE EMPIRIQUE EN CONTEXTE DIFFICILE À UNE RÉFLEXION SUR LE CONCEPT}

Chantal Amade-Escot E Patrice Venturini

Sarrazy, B. (1996). La sensibilité au contrat didactique. Rôle des arrière-plans dans la résolution de problèmes d'arithmétique au cycle trois. Thèse pour le doctorat de l'Université de Bordeaux II, non publiée, Bordeaux, France.

Schneeberger, P. (2008). Travail langagier et construction de savoirs en sciences. Les Dossiers des Sciences de l'Éducation, 20, 89-104.

Schubauer-Leoni, M.-L. (1996). Etude du contrat didactique pour des élèves en difficulté en mathématiques. Problématique didactique et/ou psychosociale. In C. Raisky, \& M. Caillot (Eds.), Au-delà des didactiques, le didactique: débats autour de concepts fédérateurs (pp.159-189). Bruxelles: De Boeck, Perspectives en éducation.

Schubauer-Leoni M.-L. (2008). La construction de la référence dans l'action conjointe professeur-élèves. In N. Wallian, M.-P. Poggi, \& M. Musard (Eds.), Co-construire des savoirs : les métiers de l'intervention dans les APSA (pp. 67-86). Besançon : Presses Universitaires de Franche Comté.

Schubauer-Leoni, M.-L., \& Leutenegger, F. (2005). Une relecture des phénomènes transpositifs à la lumière de la didactique comparée. Revue suisse des sciences de l'éducation, 27(3), 407-429.

Schubauer-Leoni, M.-L., Leutenegger, F., Ligozat, F., \& Fluckiger, A. (2007). Un modèle de l'action conjointe professeur-élèves: les phénomènes didactiques qu'il peut/doit traiter. In G. Sensevy, \& A. Mercier (Eds.), Agir ensemble. L'action didactique conjointe du professeur et des élèves (pp. 51-91). Rennes : Presses Universitaires de Rennes.

Schwartz, Y. (2001) Théories de l'action ou Rencontres de l'activité. In J.-M. Baudouin, \& J. Friedrich (Eds.), Théorie de l'action et éducation (pp. 67-91). Bruxelles : De Boeck, Raisons Educatives.

Selley, N. J. (1996). Towards a phenomenography of light and vision. International Journal of Science Education, 18(7), 837-846.

Sensevy, G. (2001). Théorie de l'action et action du professeur. In J.-M. Baudouin, \& J. Friedrich (Eds). Théories de l'action et éducation (pp. 203- 224). Bruxelles : De Boeck, Raisons Educatives.

Sensevy, G. (2007) Des catégories pour décrire et comprendre l'action didactique. In G. Sensevy, \& A. Mercier (Eds.), Agir ensemble. L'action didactique conjointe du professeur et des élèves (pp. 13-49). Rennes : Presses Universitaires de Rennes.

Sensevy, G. (2008). Le travail du professeur pour la théorie de l'action conjointe en didactique : une activité située? Recherche et Formation, 57, 39-50.

Sensevy, G., Mercier, A., \& Schubauer-Leoni, M.-L. (2000). Vers un modèle de l'action didactique du professeur. A propos de la cours à 20. Recherches en Didactique des Mathématiques, 20(3), 263-304.

Sensevy, G., \& Mercier, A. (2007). Agir ensemble. L'action didactique conjointe du professeur et des élèves. Rennes : Presses universitaires de Rennes.

Senvevy, G., \& Quilio, S. (2002). Les discours du professeur. Vers une pragmatique didactique. Revue Française de Pédagogie, 141, 47-56. 
Thevenaz-Christen, T. (2002). Milieu didactique et travail de l'élève dans une interaction maître-élève : apprendre à expliquer une règle de jeu à l'école enfantine genevoise. In P. Venturini, C. Amade-Escot, \& A. Terrisse (Eds.), Etude des pratiques effectives : l'approche des Didactiques (pp. 47-70). Grenoble: La pensée sauvage.

Thépaut A., \& Léziart, Y. (2007). De la nécessité de la prise en compte de la notion de « milieu didactique » pour l'étude des phénomènes de transmission et d'appropriation des savoirs : un exemple à propos de l'apprentissage de la passe en basket-ball. Science et Motricité, 61, 57-71.

Tsoumpélis, L. (2005). Aspects théoriques et méthodologiques de la didactique des sciences physiques. Explication et causalité dans les situations didactiques. Recherches en Didactique des Mathématiques, 25(2), 187-246.

Tiberghien, A. (1983). Revue critique sur les recherches visant à élucider le sens de la notion de lumière pour les élèves de 10 à 16 ans. In Atelier international d'été : recherche en didactique de la physique (pp.125-133). Paris, La Londe des Maures : CNRS.

Tiberghien, A., Malkoun L., Buty, C., Souassy, N., \& Mortimer E. (2007). Analyse des savoirs en jeu en classe de physique à différentes échelles de temps. In G. Sensevy, \& A. Mercier (Eds.), Agir ensemble. L'action didactique conjointe du professeur et des élèves (pp. 93-121). Rennes : Presses Universitaires de Rennes.

van Zanten, A. (2001). L'école de la périphérie. Scolarité et ségrégation en banlieue. Paris : PUF.

Venturini, P., Calmettes, B., Amade-Escot, C., \& Terrisse, A. (2007). Analyse didactique des pratiques d'enseignement de la physique d'une professeure expérimentée. Aster, 45, 211-234.

Venturini, P., \& Amade-Escot, C. (soumis). Enseigner la physique en milieu difficile : co-construction de la référence en classe dédoublée et en classe entière? Revue Suisse des Sciences de l'Éducation.

Walliser, B. (1977). Systèmes et modèles : introduction critique à l'analyse des systèmes. Paris : Seuil 